\title{
A Computationally Tractable Scheme for Simulation of the Human Pulmonary System
}

\author{
Brody H. Foy*, David Kay \\ Department of Computer Science, University of Oxford, Wolfson Building, Parks Road, \\ OX1 3QD, Oxford, UK
}

\begin{abstract}
The accurate analysis of patient lung structure and morphology in a clinical setting is one of the significant challenges in pulmonary medicine. In recent years, computational modelling techniques have been shown to help improve understanding of function-form relationships within the lungs, and of the underlying sensitivities of pulmonary function test responses to pulmonary disease. A large array of literature has been dedicated to modelling airflow and gas transfer within the lungs, and within vasculature. However, little work exists connecting the two systems together, particularly in computationally tractable ways. Within this study we outline a numerical scheme for modelling gas transport throughout the airways, pulmonary arterial network, and across the alveolar-capillary membrane. Through careful scheme design, and the application of high-performance computing structures, we show how these models can remain tractable, even when simulating to quite fine resolution. Following model development and error analysis, we apply the model to the simulation of the multiple-breath washout, a common pulmonary function test, illustrating how the choice of tracer gas may affect clinical diagnosis measures.
\end{abstract}

Keywords: Pulmonary, washout, computational, lungs, blood flow

\footnotetext{
$\Delta$

*Corresponding author

Email address: brody.foy@new.ox.ac.uk ( Brody H. Foy)
} 


\section{Introduction}

The lungs are an incredibly complex pair of organs, whose function is strongly driven by both shape and morphology. Due to this, one of the most significant challenges within pulmonary medicine is the accurate analysis of patient lung structure, and ventilation mechanics. In recent years, computational modelling approaches have been developed with the aim of better understanding the human pulmonary system, and its response to diseases.

A large array of modelling techniques have been developed [1, 2, 3, 4], often with the aim of improving understanding of pulmonary function test (PFT) responses to disease. Airway ventilation is typically simulated in either a 1D [2] or 3D-1D hybrid [5] environment, performed on virtual lungs created from histological data [6], or patient-based airway structures created from a combination CT image extraction, and algorithmic generation[1, 7]. As computational power has improved, models have increased in complexity, and been able to capture behaviours at finer resolution within the airways.

Alongside airways models, similar modelling techniques have been applied to the bloodstream, and pulmonary arterial network $[8,9,10]$. Most commonly blood flow and artery cross-sectional area profiles are simulated using the Navier-Stokes equations in a 1D setting [11], or using a hybrid 3D-1D approach [12]. Similar to ventilation models, blood flow models are typically simulated upon histological-based[13, 14] or imaging based [15] virtual vasculature structures.

While there has been a large degree of work in both modelling pulmonary flow, and modelling ventilation, there is substantially less work which connects airflow and blood flow models together, to achieve full pulmonary simulation. Detailed models of alveolar-capillary gas exchange have been derived in the literature [4], however they have typically only been applied as compartmental models, limiting many potential avenues for analysis. This in part is driven by the significant computational constraint of building a full pulmonary model. Recent work by Kang et al. [16], has outlined a way to connect mechanistic models of the pulmonary arterial network and the airways, to achieve more holistic gas transport simulation. However, this model was presented with no significant analysis of computational tractability, and thus questions remain about its feasibility for widespread implementation.

Within this study, we address the literature gap by outlining an effective and tractable model of gas transport within the conducting zone of the lungs, the pulmonary arterial network, and across the alveolar-capillary mem- 
brane. This model is primarily mechanistic, allowing for realistic simulation of blood flow, air flow, and gas transfer between the two networks. By embedding these simulations in high-performance computing architectures, we show how simulation of the human pulmonary system can be both detailed and computationally tractable. We then illustrate the potential value of such a model, by applying it to the analysis of how the choice of tracer gas may affect outputs from the multiple-breath washout, a pulmonary function test experiencing increased clinical uptake.

\section{Creation of a virtual lung and arterial structure}

To build a detailed model for simulation of the pulmonary system, we first need a physically realistic virtual representation of the lungs and pulmonary arterial network. Within this study simulations of the pulmonary system were performed using a set of 26 patient-based 1D structures ( 8 healthy subjects, 18 asthmatic subjects), taken from a set originally presented by Bordas et al. [1]. Structures were created through a combination of CT segmentation (to generation 6-10), and algorithmic airway generation within the identified lobar boundaries (to an average generation 16) [17]. Each airway tree consisted of between 30-100,000 branches, 15-50,000 of which were terminal bronchioles; each of which was subtended by a spherical acinar unit.

Construction of the arterial network was performed through modification of the patient-specific airway tree. We assume that the pulmonary arteries mimic the airway structure, with identical branching angles and ratios [6], but with resting radii (the radii at standard pressure) reduced by $75 \%$, to match healthy histological data [18]. At the distal ends of the left and right main bronchi this similarity to the airway tree ends, and subsequently we artificially create the left and right pulmonary artery, by extending a centreline directly away from the mid-point where the two inferior pulmonary arteries meet. Instead of creating an artificial pulmonary trunk, the artery network is left as two distinct structures, for the left and right lung respectively.

The veins in the network were assumed to by symmetric to the arteries, with each terminal artery connected to the associated terminal vein by a single capillary branch, with equal radius to the artery. This is illustrated in a schematic diagram in Figure 1. The capillaries were assumed to wrap around each acinar region, covering approximately $1 / 5$ of their surface area 
73 (a justification of this is given in Appendix A), leading to a capillary length

$$
l_{\mathrm{cap}}=\frac{2 \pi R_{\mathrm{acin}}^{2}}{5 R_{\mathrm{cap}}^{0}},
$$

74

where $R_{\text {acin }}$ and $R_{\text {cap }}^{0}$ are the radius of the acinar region and capillary respectively. It should be noted that given the acinar regions expand and contract over time, the length of each capillary also fluctuates in response.

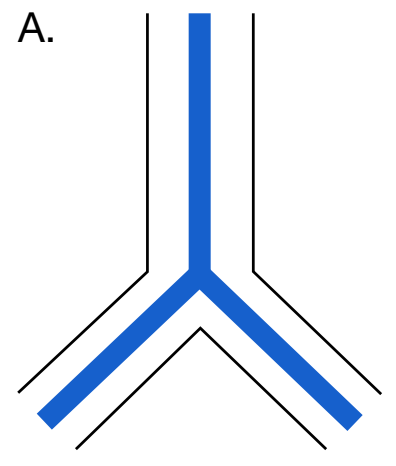

B.
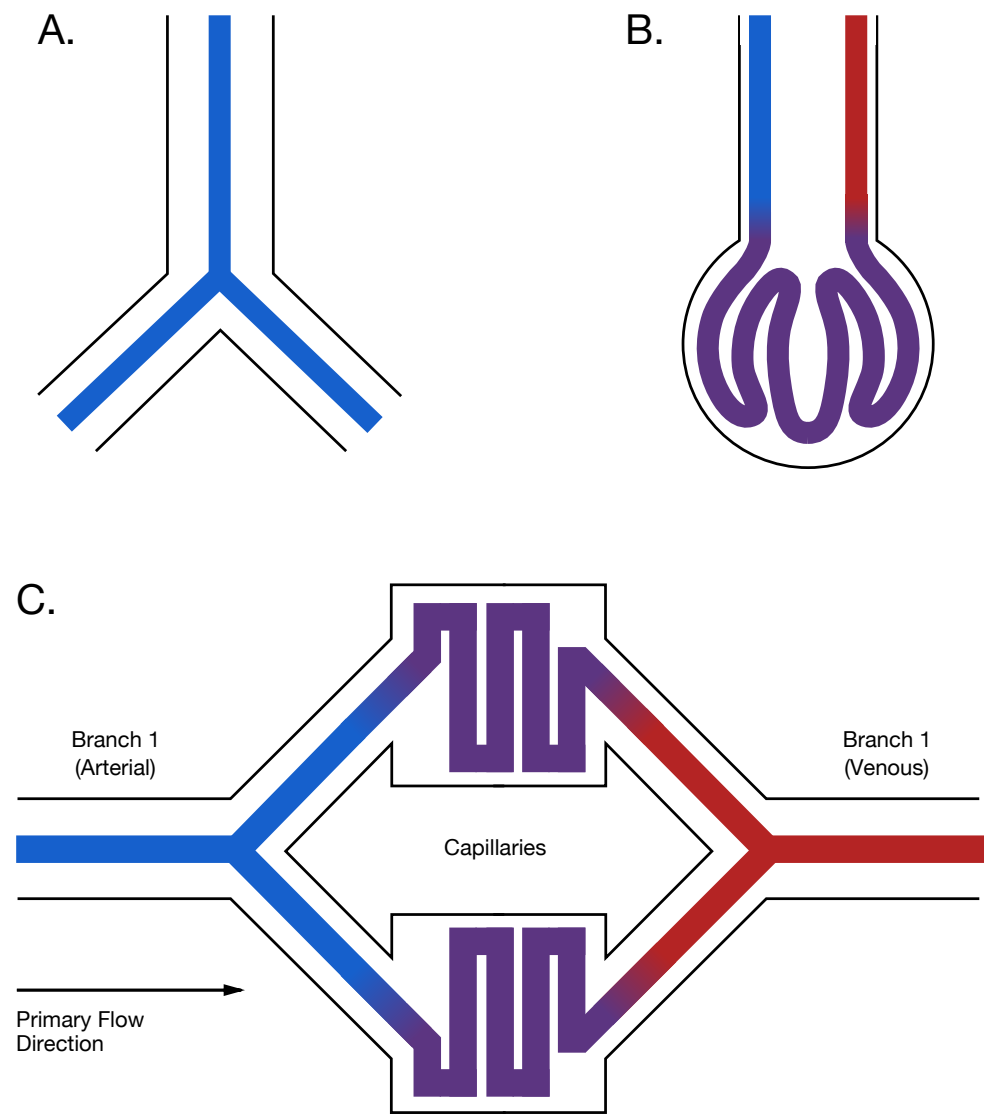

Figure 1: Diagram of the arterial network. A cross-section of the arteries against the airways can be seen (A), as well as a 2D representation of the capillaries wrapping around the acini (B). An unravelled 2D schematic of the symmetry between artery and vein networks is also given $(\mathrm{C})$. 
We note that the construction of the arterial network is based on significant simplifying assumptions about branch lengths and radii. However, the modelling techniques outlined in this study do not rely on the specific nature of the structure, and would be suitable for application to any appropriately formatted 1D representation of the lungs and associated arterial pulmonary network.

\section{Pulmonary model}

To effectively simulate gas transport throughout the pulmonary system, five different models are needed: for ventilation, blood flow, gas transport in the airways, gas transport in the bloodstream, and gas transfer across the alveolar-capillary membrane. Within this section we detail each model and its numerical implementation.

\subsection{Ventilation model}

Detailed explanations of the ventilation and airways gas transfer models have appeared in the prior work of Foy et al. [2, 19, 20]. In brief, the flow rate $Q$ within each airway is assumed to be driven by the pressure gradient $\Delta P$ over the branch. Each terminal bronchiole is subtended by a spherical acinar region $V_{\text {acin }}$, whose expansion and contraction is proportional to the terminal bronchiole flow rate, and constrained by local tissue compliance $\mathbb{C}$, and the pressure difference from the acinus to the pleural cavity. This is expressed by the equation system

$$
\begin{aligned}
\Delta P & =R(Q) Q, & & \text { for each branch, } \\
Q_{\text {upper }} & =\sum Q_{\text {lower }}, & & \text { for each bifurcation, } \\
\frac{d}{d t} V_{\text {acin }} & =Q, & & \text { for each terminal branch, } \\
P_{T}-P_{p l}(t) & =\frac{1}{\mathbb{C}} V_{\text {acin }}, & & \text { for each terminal branch. }
\end{aligned}
$$

where $R(Q)$ is Poiseuille branch resistance, with a modification for energy dissipation [21], $P_{T}$ is the pressure at the distal end of the terminal branch, and $P_{p l}$ is the pleural pressure. A diagram of the ventilation domain is given in Figure 2. 


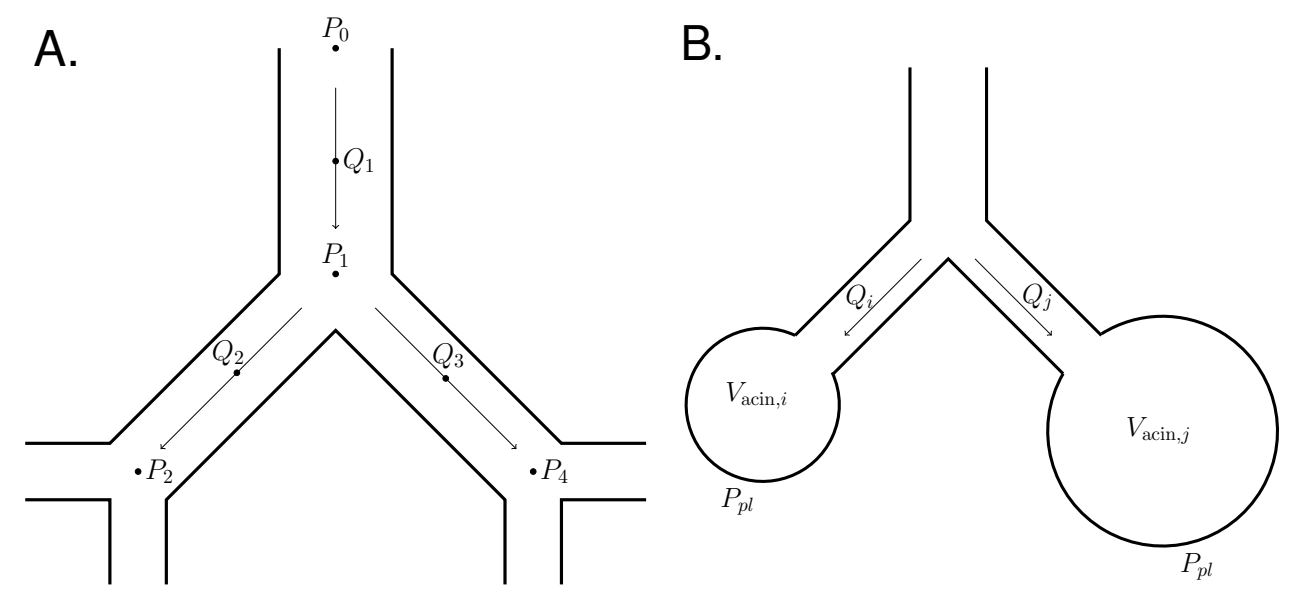

Figure 2: Diagram of the ventilation model domain. An idealised 2D cross-section of the model domain, showing branch bifurcations, flow rates and pressures $(\mathrm{A})$, and the connection of terminal bronchioles to acinar regions (B).

The pleural pressure is chosen to enforce the desired breathing pattern and frequency (typically sinusoidal for tidal breathing), with a linear gravitational gradient on its range (see Foy et al. [2] for more details).

System (2)-(5) was implemented numerically using a Backward Euler discretisation for the temporal derivative, leading to a system of the form

$$
\mathbf{F}(\mathbf{x})=\mathbf{0},
$$

at each time step, where $\mathbf{x}$ is the collection of pressure, flow and volume variables. This system is solved using vector Newton iterations, of the form

$$
\mathbf{x}=\mathbf{x}-\mathcal{J}^{-1} \mathbf{F}(\mathbf{x}),
$$

until $\|\mathbf{F}(\mathbf{x})\|_{2}$ falls below a desired tolerance.

As the majority of the Jacobian $(\mathcal{J})$ is constant, it can be efficiently formed directly, and inverted using an LU factorisation following approximateminimum-degree reordering.

\subsection{Airways gas transport model}

In the absence of bloodstream interactions, the ventilation model outlined above is used to drive a gas transport model in the airways, first proposed 
by Paiva [22]:

$$
\frac{\partial C}{\partial t}=D \frac{\partial^{2} C}{\partial x^{2}}+\frac{D}{A} \frac{\partial A}{\partial x} \frac{\partial C}{\partial x}-u \frac{\partial C}{\partial x}
$$

where $C(x, t)$ is the gas concentration at position $x$ and time $t, A$ the airway cross-sectional area, $u=Q / A$ the air velocity, and $D$ the diffusion coefficient for the gas in air.

To implement this model numerically, we first discretise the domain into a series of $N$ nodes, with corresponding concentration values $C_{i}(i=1, \ldots, N)$. Multiple nodes are placed on each branch, a single node is placed at the centre of each acinar region, and a single node is placed slightly proximal to each bifurcation, as illustrated in Figure 3.
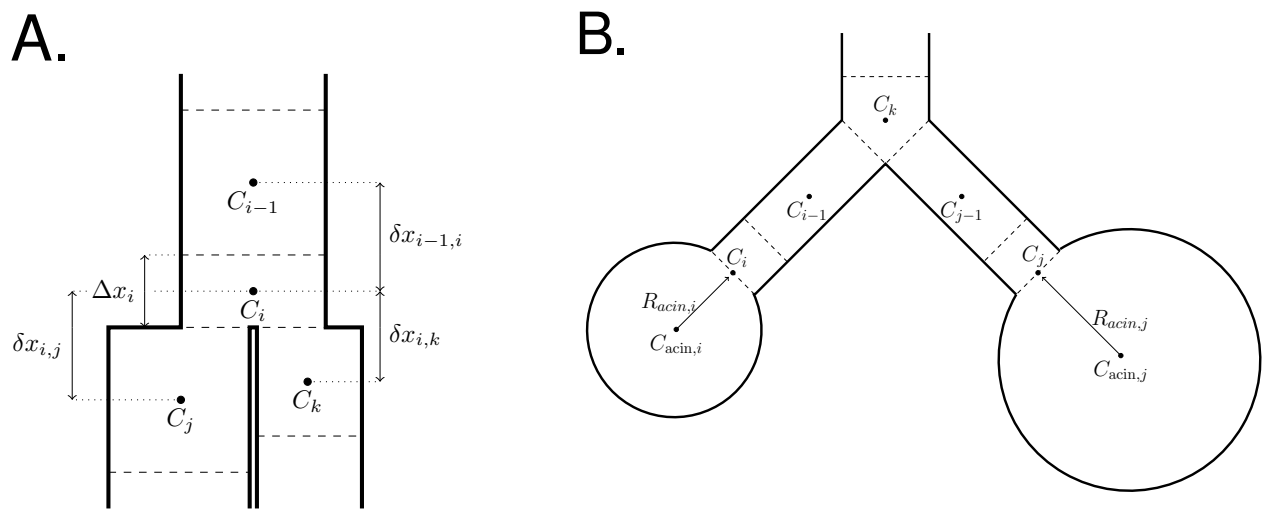

Figure 3: Diagram of the airways gas transport model domain. An idealised crosssection of the model domain, showing concentration nodes and finite difference boundaries in the branches $(\mathrm{A})$, and the acinar regions $(\mathrm{B})$.

The gas transport model is then implemented numerically using a finite difference scheme, originally outlined by Dutrieue et al. [23]. For each node 
at a bifurcation, and considering the notation in Figure 3, we have

$$
\begin{array}{r}
\frac{d C_{i}}{d t}=\frac{D}{\Delta x_{i}}\left(\frac{1}{A_{j}+A_{k}}\left\{\frac{\left[C_{j}-C_{i}\right] A_{j}}{\delta x_{i, j}}+\frac{\left[C_{k}-C_{i}\right] A_{k}}{\delta x_{i, k}}\right\}-\frac{C_{i}-C_{i-1}}{\delta x_{i-1, i}}\right) \\
+\frac{D}{2 A_{i}}\left(\frac{\left\{A_{j}-A_{i} \frac{A_{j}}{A_{j}+A_{k}}\right\}\left[C_{j}-C_{i}\right]}{\delta x_{i, j}^{2}}+\frac{\left\{A_{k}-A_{i} \frac{A_{k}}{A_{j}+A_{k}}\right\}\left[C_{k}-C_{i}\right]}{\delta x_{i, k}^{2}}\right) \\
+\left\{\begin{array}{l}
\frac{u_{i} C_{i}-u_{i-1} C_{i-1}}{\Delta x_{i}}, \quad \text { during inspiration } \\
\frac{u_{j} C_{j}+u_{k} C_{k}-u_{i} C_{i}}{\Delta x_{i}}, \text { during expiration. }
\end{array}\right.
\end{array}
$$

A similar formula can be derived for nodes within a branch, by treating it as a single branch bifurcation, and setting appropriate terms to zero.

At the tracheal boundary, the system is convection-dominated meaning

$$
\frac{\partial C}{\partial \mathbf{n}}= \begin{cases}u_{1} C_{\mathrm{ext}}, & \text { during inspiration } \\ u_{1} C_{1}, & \text { during expiration }\end{cases}
$$

where $\mathbf{n}$ is the normal vector outwards from the boundary, and $C_{\text {ext }}$ is the external gas concentration.

At each terminal bronchiole boundary gas transfer occurs between the acinus and the bronchiole. To account for this, we enforce the boundary condition

$$
\frac{\partial C_{i}}{\partial \mathbf{n}}=D \frac{C_{\mathrm{acin}, j}-C_{i}}{R_{\mathrm{acin}, j}}- \begin{cases}u_{i} C_{i}, & u_{i}>0 \\ u_{i} C_{\mathrm{acin}, j}, & u_{i} \leq 0\end{cases}
$$

where $R_{\mathrm{acin}, j}$ is the radius of the acinar region, calculated as

$$
R_{\mathrm{acin}, j}=\left(\frac{3}{4 \pi} V_{\mathrm{acin}, j}\right)^{1 / 3}
$$

Gas concentrations in each acinus fluctuate accordingly to the gas transfer 
from the terminal bronchiole, and changes in acinar volume, meaning

$$
\frac{d V_{\mathrm{acin}, j} C_{\mathrm{acin}, j}}{d t}= \begin{cases}Q_{i} C_{i}-D A_{i}\left(C_{\mathrm{acin}, j}-C_{i}\right) / R_{\mathrm{acin}, j}, & Q_{i}>0 \\ -D A_{i}\left(C_{\mathrm{acin}, j}-C_{i}\right) / R_{\mathrm{acin}, j}, & Q_{i} \leq 0\end{cases}
$$

The total model for gas transport in the airways is solved using a combination of Euler methods and operator splitting. Firstly, all temporal derivatives in the airways are discretised using a Backward Euler method. This allows for airway gas concentrations to be updated through solution of a matrix problem

$$
A \mathbf{C}^{n+1}=\mathbf{C}^{n}+\mathbf{f}\left(\mathbf{C}^{n}, \mathbf{C}_{\text {acin }}^{n}\right)
$$

where $A$ is a convection-diffusion matrix, $\mathbf{C}^{n}$ and $\mathbf{C}_{\text {acin }}^{n}$ are the airway and acinus concentrations at time step $n$, and $\mathbf{f}$ accounts for boundary conditions between terminal bronchioles and the associated acini. The matrix inversion is solved using a standard LU factorisation, after application of approximateminimum-degree reordering.

Following the calculation of $\mathbf{C}^{n+1}$, the updated acinar concentrations $\left(\mathbf{C}_{\text {acin }}^{n+1}\right)$ are calculated by applying a Forward Euler discretisation to Equation (8).

\subsection{Blood flow model}

To model blood flow, we build upon the work of Olufsen et al. [11]. By considering experimental data, Olufsen [24] showed that there was an apparent function relationship between arterial wall thickness, vessel radius and vessel stiffness, such that

$$
\frac{E h}{R_{b}^{0}}=k_{1} \exp \left(k_{2} R_{b}^{0}\right)+k_{3},
$$

where $k_{1}, k_{2}$ and $k_{3}$ are fitting constants, given in Table 1 , and $R_{b}^{0}$ is the arterial radius under standard pressure.

Given this, we assume a linear stress-strain relationship between artery cross-sectional area $\left(A_{b}\right)$, and pressure, such that

$$
P_{b}=\frac{4}{3} \frac{E h}{R_{b}^{0}}\left(1-\sqrt{\frac{A_{b}^{0}}{A_{b}}}\right)=f\left(R_{b}^{0}\right)\left(1-\sqrt{\frac{A_{b}^{0}}{A_{b}}}\right),
$$


where $A_{b}$ is the cross-sectional area of the vessel, and $A_{b}^{0}$ is the cross-sectional area at standard pressure. We combine this model with a 1D Navier Stokes equation, meaning

$$
\begin{aligned}
\frac{\partial A_{b}}{\partial t}+\frac{\partial Q_{b}}{\partial x} & =0 \\
\frac{\partial Q_{b}}{\partial t}+\frac{\partial}{\partial x}\left(\alpha \frac{Q_{b}^{2}}{A_{b}}+B\right) & =-\frac{2 \pi \nu_{b} Q_{b} R_{b}}{\delta A_{b}}+\frac{\partial B}{\partial R_{b}^{0}} \frac{d R_{b}^{0}}{d x}
\end{aligned}
$$

where $Q_{b}$ is the flow rate, $\nu_{b}$ is the dynamic viscosity of blood, $\delta$ is the boundary layer thickness and $\rho_{b}$ is the density of blood. The term $B=f \sqrt{A_{b}^{0} A_{b}} / \rho_{b}$ is an artificial term constructed to allow the equations to be written in conservative form. The parameter $\alpha$ is a correction factor, presented by Sherwin et al. [25], which accounts for the shape of the uni-directional velocity profile. Within this study, for simplicity we take $\alpha=1$, corresponding to a flat profile. In Appendix B, we compare flow profiles throughout the pulmonary system using $\alpha=1.1$, showing strong concordance.

To simplify manipulations of equations (9)-(10), we introduce the notation

$$
\mathbf{U}=\left[\begin{array}{c}
A_{b} \\
Q_{b}
\end{array}\right], \mathbf{T}=\left[\begin{array}{c}
\mathcal{T}_{1} \\
\mathcal{T}_{2}
\end{array}\right]=\left[\begin{array}{c}
Q_{b} \\
\frac{Q_{b}^{2}}{A_{b}}+B
\end{array}\right], \mathbf{S}=\left[\begin{array}{c}
\mathcal{S}_{1} \\
\mathcal{S}_{2}
\end{array}\right]=\left[\begin{array}{c}
0 \\
-\frac{2 \pi \nu Q_{b} R_{b}}{\delta A_{b}}+\frac{\partial B}{\partial R_{b}^{0}} \frac{\partial R_{b}^{0}}{\partial x}
\end{array}\right],
$$

meaning the system can be written as

$$
\frac{\partial}{\partial t} \mathbf{U}+\frac{\partial}{\partial x} \mathbf{T}=\mathbf{S}
$$

We solve this system numerically using a two-step Lax-Wendroff Method. First each arterial branch is discretise into $M$ evenly spaced nodes (with spacing $\Delta x$ ), at $N$ evenly spaced points in time (with time step $\Delta t$ ). We denote the value of $U_{b}$ at node $M$, and time step $n$ as $U_{M}^{n}$, and define intermediate step values as

$$
\mathbf{U}_{j}^{n+1 / 2}=\frac{\mathbf{U}_{j+1 / 2}^{n}+\mathbf{U}_{j-1 / 2}^{n}}{2}+\frac{\Delta t}{2}\left(-\frac{\mathbf{T}_{j+1 / 2}^{n}-\mathbf{T}_{j-1 / 2}^{n}}{\Delta x}+\frac{\mathbf{S}_{j+1 / 2}^{n}+\mathbf{S}_{j-1 / 2}^{n}}{2}\right)
$$

for $j=m \pm 1 / 2$. The full step solution is then defined from the intermediate 
step values as

$$
\mathbf{U}_{m}^{n+1}=\mathbf{U}_{m}^{n}-\frac{\Delta t}{\Delta x}\left(\mathbf{T}_{m+1 / 2}^{n+1 / 2}-\mathbf{T}_{m-1 / 2}^{n+1 / 2}\right)+\frac{\Delta t}{2}\left(\mathbf{S}_{m+1 / 2}^{n+1 / 2}+\mathbf{S}_{m-1 / 2}^{n+1 / 2}\right) .
$$

\subsubsection{Bifurcation conditions}

To ensure conservation each bifurcation is decomposed into a parent node and daughter nodes, as illustrated in Figure 4. At each branch bifurcation, continuity of pressure, and conservation of flow gives

$$
\begin{aligned}
Q_{b, j}^{p, M} & =Q_{b, 0}^{d_{1}, j}+Q_{b, 0}^{d_{2}, j}, \\
P_{b, M}^{p, j} & =P_{b, 0}^{d_{1}, j}=P_{b, 0}^{d_{2}, j},
\end{aligned}
$$

where $p, d_{1}$ and $d_{2}$ denote the parent and daughter nodes of the bifurcation, and $j=n+1 / 2, n+1$.

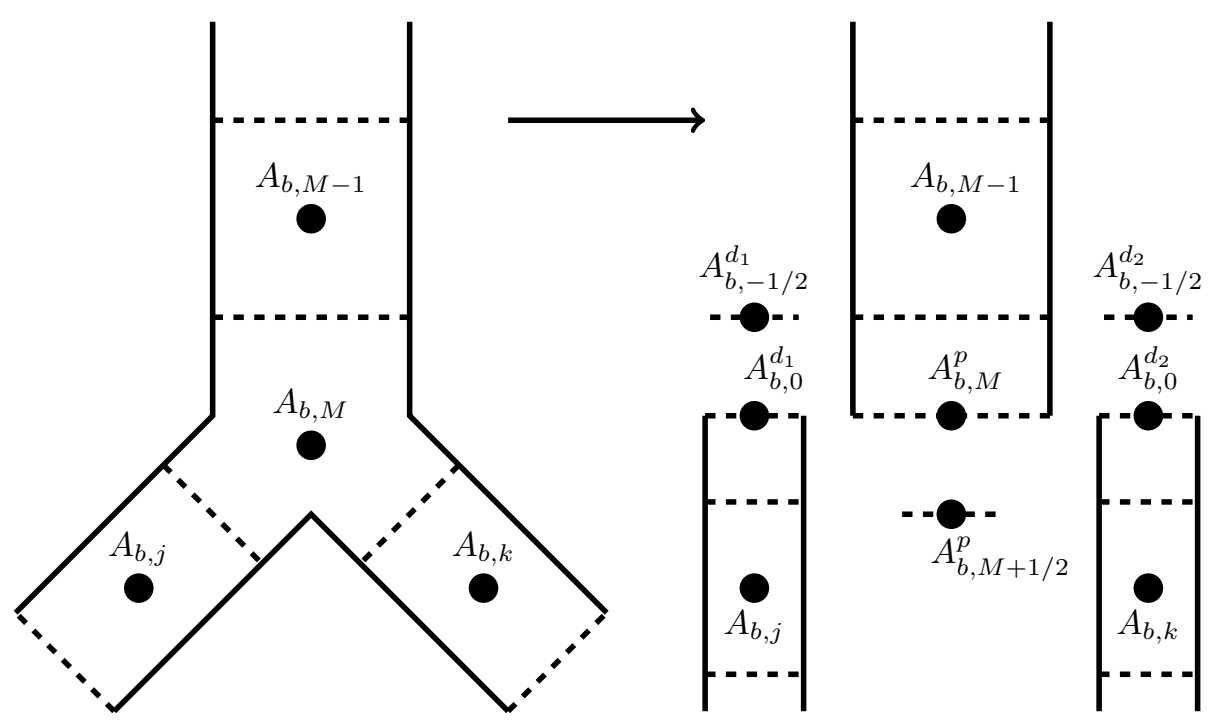

Figure 4: Diagram of a 3-branch bifurcation decomposition. The diagram shows a bifurcation before (left) and after (right) decomposition. New nodes $d_{1}$ and $d_{2}$ are created, alongside ghost node values $A_{b,-1 / 2}^{d_{1}}, A_{b,-1 / 2}^{d_{2}}$, and $A_{b, M+1 / 2}^{p}$. Similar nodes are also created for all corresponding $Q_{b}$ values.

${ }_{11}$ Given $P_{b}$ is a function of $A_{b}$, the pressure continuity equation can be 
re-written as

$$
f_{M}^{p}\left(1-\sqrt{\frac{A_{b, M}^{p, 0}}{A_{b, M}^{p, n+1}}}\right)=f_{0}^{d_{1}}\left(1-\sqrt{\frac{A_{b, 0}^{d_{1}, 0}}{A_{b, 0}^{d_{1}, n+1}}}\right),
$$

and similar for $d_{2}$. These six continuity equations can be taken alongside the Lax-Wendroff scheme equations

$$
\begin{aligned}
& A_{b, m}^{i, n+1}=A_{b, m}^{i, n}+\frac{\Delta t}{\Delta x}\left(\mathcal{T}_{1, m+1 / 2}^{i, n+1 / 2}+\mathcal{T}_{1, m-1 / 2}^{i, n+1 / 2}\right) \\
& Q_{b, m}^{i, n+1}=Q_{b, m}^{i, n}+\frac{\Delta t}{\Delta x}\left(\mathcal{T}_{2, m+1 / 2}^{i, n+1 / 2}-\mathcal{T}_{2, m-1 / 2}^{i, n+1 / 2}\right)+\frac{\Delta t}{2}\left(\mathcal{S}_{2, m+1 / 2}^{i, n+1 / 2}+\mathcal{S}_{2, m-1 / 2}^{i, n+1 / 2}\right),
\end{aligned}
$$

173

where $i=p, d_{1}, d_{2}$, and $m=M$ for $p$, and $m=0$ for $d_{1}$ and $d_{2}$.

This gives 12 equations, which govern the 12 state variables $Q_{b, m}^{i, j}, A_{b, m}^{i, j}$ for $i=p, d_{1}, d_{2}$, and $j=n, n+1$. However, these equations also require 6 variables which exist only as ghost nodes: $A_{b, M+1 / 2}^{p, n+1 / 2}, A_{b,-1 / 2}^{d_{1}, n+1 / 2}, A_{b,-1 / 2}^{d_{2}, n+1 / 2}$, and similar for $Q_{b}$. This requires 6 more equations to close the system, which are created by defining the ghost points through averaging, such that

$$
\begin{aligned}
Q_{b, m}^{i, n+1 / 2} & =\frac{Q_{b, m-1 / 2}^{i, n+1 / 2}+Q_{b, m+1 / 2}^{i, n+1 / 2}}{2}, \\
A_{b, m}^{i, n+1 / 2} & =\frac{A_{b, m-1 / 2}^{i, n+1 / 2}+A_{b, m+1 / 2}^{i, n+1 / 2}}{2},
\end{aligned}
$$

where $i=p, d_{1}, d_{2}$, and $m=M$ for $p$ and $m=0$ for $d_{1}$ and $d_{2}$.

This gives rise to a closed non-linear system of 18 equations. However, many of the equations are linear, meaning the system can actually be decomposed into two sets of smaller equation systems (more detail of this is given in Appendix C).

The system outlined above is for a bifurcation with one parent and two daughters. However, similar equation systems follow for other bifurcation types (e.g. two parents one daughter, in the venous network, or one parent and one daughter at the artery-capillary join). 


\subsubsection{Inlet boundary condition}

At the two inlet boundaries (the left and right pulmonary arteries), a flow rate boundary condition is prescribed such that

$$
\begin{aligned}
Q_{b}(0, t) & =\frac{\bar{Q}_{b} t}{\tau} \exp \left(\frac{1}{2}\left(1-t^{2} / \tau^{2}\right)\right), & & 0 \leq t<H_{T}, \\
Q_{b}\left(0, t+j H_{T}\right) & =Q_{b}(0, t), & j & =1,2,3, \ldots
\end{aligned}
$$

where $\bar{Q}_{b}$ is the maximum flow, $\tau$ is the time of maximum flow, and $H_{T}$ is the heartbeat period.

We calculate the inflow boundary cross-sectional area, by first introducing a ghost point $Q_{b,-1 / 2}^{n+1}$ which satisfies

$$
Q_{b, 0}^{n+1 / 2}=\frac{Q_{b,-1 / 2}^{n+1 / 2}+Q_{b, 1 / 2}^{n+1 / 2}}{2}
$$

where $Q_{b, 0}^{n+1 / 2}$ is the inflow rate, evaluated at time step $n+1 / 2$. Using this ghost point, the inflow area $A_{b}^{0}$ can be calculated as

$$
A_{b, 0}^{n+1}=A_{b, 0}^{n}-\frac{\Delta t}{\Delta x}\left(\mathcal{T}_{1,1 / 2}^{n+1 / 2}-\mathcal{T}_{1,-1 / 2}^{n+1 / 2}\right)+\frac{\Delta t}{2}\left(\mathcal{S}_{1,1 / 2}^{n+1 / 2}+\mathcal{S}_{1,-1 / 2}^{n+1 / 2}\right),
$$

where $\mathcal{T}_{1,-1 / 2}^{n+1 / 2}=Q_{b,-1 / 2}^{n+1 / 2}$ and $\mathcal{S}_{1,-1 / 2}^{n+1 / 2}=0$

\subsubsection{Outlet boundary condition}

At each outlet boundary, we model the out of domain resistance and compliance using a 3-element Windkessel model [26] with parameters $\mathcal{R}_{1}, \mathcal{R}_{2}$, and $\mathcal{C}_{t}$. This leads to the boundary model

$$
\frac{\partial P_{b}}{\partial t}=\mathcal{R}_{1} \frac{\partial Q_{b}}{\partial t}-\frac{P_{b}}{\mathcal{R}_{2} \mathcal{C}_{T}}+\frac{Q_{b}\left(\mathcal{R}_{1}+\mathcal{R}_{2}\right)}{\mathcal{R}_{2} \mathcal{C}_{T}}
$$

which is discretised using a Forward Euler method, as

$$
P_{b, M}^{n+1}=P_{b, M}^{n}+\mathcal{R}_{1}\left(Q_{b, M}^{n+1}-Q_{b, M}^{n}\right)+\Delta t\left(-\frac{P_{b, M}^{n}}{\mathcal{R}_{2} \mathcal{C}_{T}}+\frac{Q_{b, M}^{n}\left(\mathcal{R}_{1}+\mathcal{R}_{2}\right)}{\mathcal{R}_{2} \mathcal{C}_{T}}\right)
$$


where

$$
P_{b, M}^{n+1}=\frac{f_{M}}{\rho_{b}}\left(1-\sqrt{\frac{A_{b, M}^{0}}{A_{b, M}^{n+1}}}\right) .
$$

A second equation is constructed through an upwind discretisation of Equation (9):

$$
A_{b, M}^{n+1}=A_{b, M}^{n}-\frac{\Delta t}{\Delta x}\left(Q_{b, M}^{n+1}-Q_{b, M-1}^{n+1}\right) .
$$

These two equations are solved iteratively using Jacobi's method until a convergent solution is reached (to a desired tolerance).

\subsubsection{Computational implementation}

The model outlined in the previous section is both highly non-linear and extremely large. At a minimum, each branch in the system requires three nodes (one at each end, and a central node), both with two unknown variables $\left(A_{b}, Q_{b}\right)$. This means at the lowest resolution for a full conducting airway zone structure (approximately 100,000 branches, 50,000 of which are terminal), the corresponding vasculature system has 250,000 arterial branches, and thus 1,500,000 variables. While the Lax-Wendroff scheme is explicit, due to conservation constraints each bifurcation (of which there are approximately 200,000) is constrained by 18 non-linear equations. In Appendix C we illustrate how the computational challenges of the bifurcation conditions can be reduced through careful algebraic manipulation; however, even with these simplifications, the resulting system is incredibly large and computationally intensive.

This is further exacerbated by the time step constraints imposed by the explicit solution scheme. Assuming a uniform spatial discretisation over each branch, it can be shown that the Lax-Wendroff method is stable [11] if the associated Courant Friedrichs Lewy (CFL) condition is fulfilled:

$$
\frac{\Delta t}{\Delta x} \leq\left|\frac{Q_{b}}{A_{b}} \pm c\right|^{-1}
$$

for both choices of sign, where

$$
c=\sqrt{\frac{A_{b}}{\rho_{b}} \frac{\partial P_{b}}{\partial A}},
$$




\begin{tabular}{|c|c|c|c|}
\hline Parameter & Value & Interpretation & Value taken from: \\
\hline $\mathcal{C}_{T}$ & $1.3 \times 10^{-6} \mathrm{~cm}^{4} \mathrm{~s}^{2} / \mathrm{g}$ & Windkessel parameter & Olufsen [24] \\
\hline$D_{H e}$ & $0.803 \mathrm{~atm} . \mathrm{cm}^{2} \cdot \mathrm{s}-1$ & Diffusion rate for He in air & Marrero et al. [27] \\
\hline$D_{S F_{6}}$ & $0.102 \mathrm{~atm} \cdot \mathrm{cm}^{2} \cdot \mathrm{s}^{-1}$ & Diffusion rate for $\mathrm{SF}_{6}$ in air & Marrero et al. [27] \\
\hline$D_{N_{2}}$ & $0.2 \mathrm{~atm} . \mathrm{cm}^{2} \cdot \mathrm{s}-1$ & Diffusion rate for $\mathrm{N}_{2}$ in air & Marrero et al. [27] \\
\hline $\mathcal{D}_{\text {cap }}$ & $5 \times 10^{-4} \mathrm{~L} / \mathrm{s} / \mathrm{mmHg}$ & Diffusing capacity of $N_{2}$ & $\begin{array}{l}\text { Average for } \mathrm{O}_{2} \text { and } \\
\mathrm{CO}_{2} \text { Ben-Tal }[4]\end{array}$ \\
\hline$\delta$ & $0.1 \mathrm{~cm}$ & Artery boundary layer size & Olufsen et al. [11] \\
\hline$H_{T}$ & 1s & Heartbeat period & Standard value \\
\hline$k_{1}$ & $2 \times 10^{7} \mathrm{~g} / \mathrm{s}^{2} \mathrm{~cm}$ & Fit parameter for $f\left(R_{b}^{0}\right)$ & Olufsen [24] \\
\hline$k_{2}$ & $-22.53 / \mathrm{cm}$ & Fit parameter for $f\left(R_{b}^{0}\right)$ & Olufsen [24] \\
\hline$k_{3}$ & $8.65 \times 10^{5} \mathrm{~g} / \mathrm{s}^{2} \mathrm{~cm}$ & Fit parameter for $f\left(R_{b}^{0}\right)$ & Olufsen $[24]$ \\
\hline$\rho_{b}$ & $1060 \mathrm{~kg} / \mathrm{m}^{3}$ & Density of blood & Saladin and Porth [28] \\
\hline $\bar{Q}_{b}$ & $20 \mathrm{~cm}^{3} / \mathrm{s}$ & Maximum inlet flow rate & Olufsen [24] \\
\hline $\mathcal{R}_{1}$ & $25300 \mathrm{~g} /\left(\mathrm{s} \mathrm{cm}^{4}\right)$ & Windkessel parameter & Olufsen $[24]$ \\
\hline $\mathcal{R}_{2}$ & $13900 \mathrm{~g} /\left(\mathrm{s} \mathrm{cm}^{4}\right)$ & Windkessel parameter & Olufsen $[24]$ \\
\hline$\sigma_{b}$ & $1.7 \times 10^{-5} / \mathrm{mmHg}$ & Solubility of $N_{2}$ in blood & Sander $[29]$ \\
\hline$\tau$ & $0.1 \mathrm{~s}$ & $\begin{array}{l}\text { Time of peak inlet flow } \\
\text { (during each heartbeat) }\end{array}$ & Olufsen [24] \\
\hline$t_{\text {circulation }}$ & $60 \mathrm{~s}$ & $\begin{array}{l}\text { Average time taken for } \\
\text { blood to circulate the body }\end{array}$ & Katz $[30]$ \\
\hline$\nu_{b}$ & $0.046 \mathrm{~cm}^{2} / \mathrm{s}$ & Dynamic viscosity of blood & Olufsen et al. [11] \\
\hline
\end{tabular}

Table 1: Pulmonary model parameters

is the pulse wave speed.

In standard implementation on a full conducting zone structure, we have found this leads to an average constraint condition of

$$
\Delta t \leq 10^{-6}
$$


For non-trivial simulations of ventilation and gas transfer, a stable blood profile is needed for at least the duration of a breathing cycle. In practice, this means blood flow needs to be simulated for a duration of 3 breathing cycles (to allow for solution stabilisation), equating to 12 seconds at a standard breathing rate. Thus, total solution of the system requires explicit iteration of a system of approximately 1,500,000 variables more than $10^{7}$ times with the Lax-Wendroff scheme.

In a standard CPU architecture this would be computationally intractable, with estimated runtime being greater than 1 year per profile. However, given that the solution scheme is explicit, the calculations (within each time step) are highly parallelisable. To exploit this structure, we solve the blood flow model in a GPU environment. A standard GPU (as of 2018) has between 1000-4000 cores. This means that the incorporation of GPU computing could be expected to increase runtime by an order of $10^{3}-10^{4}$ (for a sufficiently large system). This was one of the primary motivations for the use of an explicit solution scheme. The explicit scheme introduces a harsh time constraint penalty, but the use of high-performance computing greatly offsets this effect. Equally, given the highly non-linear nature of the system (particularly the continuity constraints across each bifurcation), for accurate solution an implicit scheme would also be expected to require quite a small time step. However, an implicit scheme would not be able to be implemented in a GPU environment with the same degree of ease or effectiveness as the explicit scheme.

GPU implementation of the blood flow model was performed using gpuArray structures within MATLAB's Parallel Computing Toolbox. All simulations of blood flow were computed using an Alienware 17 R2 laptop (i7 processor, NVIDIA GTX970 GPU).

\subsection{Blood gas transport model}

Following calculation of bloodflow nad arterial cross-sectional area, these profiles are used to drive the convective transport equation

$$
\frac{\partial A_{b} C_{b}}{\partial t}=\frac{Q_{b}}{A_{b}} \frac{\partial A_{b} C_{b}}{\partial x},
$$

where $C_{b}$ is the gas concentration.

It should be noted that while $A_{b}$ and $Q_{b}$ appear in Equation (20), they can be solved independently of $C_{b}$. Given this, the model can be implemented 
numerically using a similar approach to that given in Equation (7), setting $D=0$.

At each outflow branch, during outward flow, concentration is removed from the branch, proportional to the flow rate, meaning

$$
\frac{\partial C_{b}}{\partial \mathbf{n}}=\frac{Q_{b}}{A_{b}} C_{b}
$$

where $\mathbf{n}$ is the outward normal vector to the boundary. During inward flow, it is assumed that no concentration of gas re-enters the system, such that

$$
\frac{\partial C_{b}}{\partial \mathbf{n}}=0
$$

At each inflow boundary, flow is only positive (due to the prescribed inflow, given in Equation (19). Thus, there is one inflow condition of the form

$$
\frac{\partial C_{b}}{\partial \mathbf{n}}=\frac{Q_{b}}{A_{b}} C_{\mathrm{in}}
$$

where $C_{\text {in }}$ is the upstream gas concentration.

To create a closed system, gas which exits the pulmonary veins is brought back into the pulmonary arteries, via the equation

$$
C_{\text {in }}(t)=\bar{C}_{\text {out }}\left(t-t_{\text {circulation }}\right),
$$

where $\bar{C}_{\text {out }}$ is the averaging outflow gas concentration, and $t_{\text {circulation }}$ is the average time it takes the gas to circulate the body (to get from the outflow point back to the inlet). Clearly this is a simplified model that could be updated to incorporate other factors such as tissue absorption, or chemical reactions within the body.

\subsection{Alveolar-capillary transport model}

To close the system, we link the two gas transport models, with a model for transfer across the alveolar-capillary membrane. Transport rates are as- 
sumed proportional to the partial pressure gradient, such that [4]:

$$
\begin{aligned}
\frac{\partial V_{\text {acin }} C_{\text {acin }}}{\partial t} & =\mathcal{D}_{\text {cap }}\left(P_{p, b}-P_{p, \text { acin }}\right) \\
\frac{\partial V_{b} C_{b}}{\partial t} & =-\mathcal{D}_{\text {cap }}\left(P_{p, b}-P_{p, \text { acin }}\right),
\end{aligned}
$$

where $C_{b}$ is the concentration in the centre of the capillary, $C_{\text {acin }}$ is the concentration in the associated acinar region, and $\mathcal{D}_{\text {cap }}$ is the diffusing capacity (not to be confused with diffusion rate) of the gas across the alveolar-capillary membrane. $P_{p, b}$ and $P_{p, \text { acin }}$ are the partial pressures of the gas in blood and the acinus respectively, calculated as

$$
P_{p, b}=\frac{1}{\sigma_{b}} C_{b}
$$

$$
P_{p, \text { acin }}=P_{\text {acin }} C_{\text {acin }},
$$

where $\sigma_{b}$ is the solubility of the gas in blood, and $P_{\text {acin }}$ is the gas pressure inside the acinus, which is the total pressure, minus the water vapour pressure (typically $47 \mathrm{mmHg}$ ).

\subsection{Total model}

Combining the models outlined in the previous sections we can achieve simulation of gas transport throughout the entire pulmonary system. The process for this is described in Figure 5. Firstly, the ventilation profile and associated acinar volumes are calculated using System (2)-(5). The acinar volumes are then used to calculate capillary lengths with Equation (1), meaning the blood flow profile and arterial areas can be simulated through System (9)-(10). Once the ventilation and blood flow distributions are calculated, gas transfer is simulated using Equation (6) for the airways and Equation (20) for the bloodstream. Both of these equations are discretised using the same time step size, and at each time step are solved independently of each other. Between time steps, System (22)-(23) is used to account for gas transfer across the alveolar-capillary membrane.

Under an assumption of steady breathing and heart rate, ventilation and blood flow profiles are simulated over consecutive breathing cycles until stability (within 1\%) is reached (typically taking 3 periods). These profiles are then repeatedly used to simulate gas transfer for as long as necessary. While 


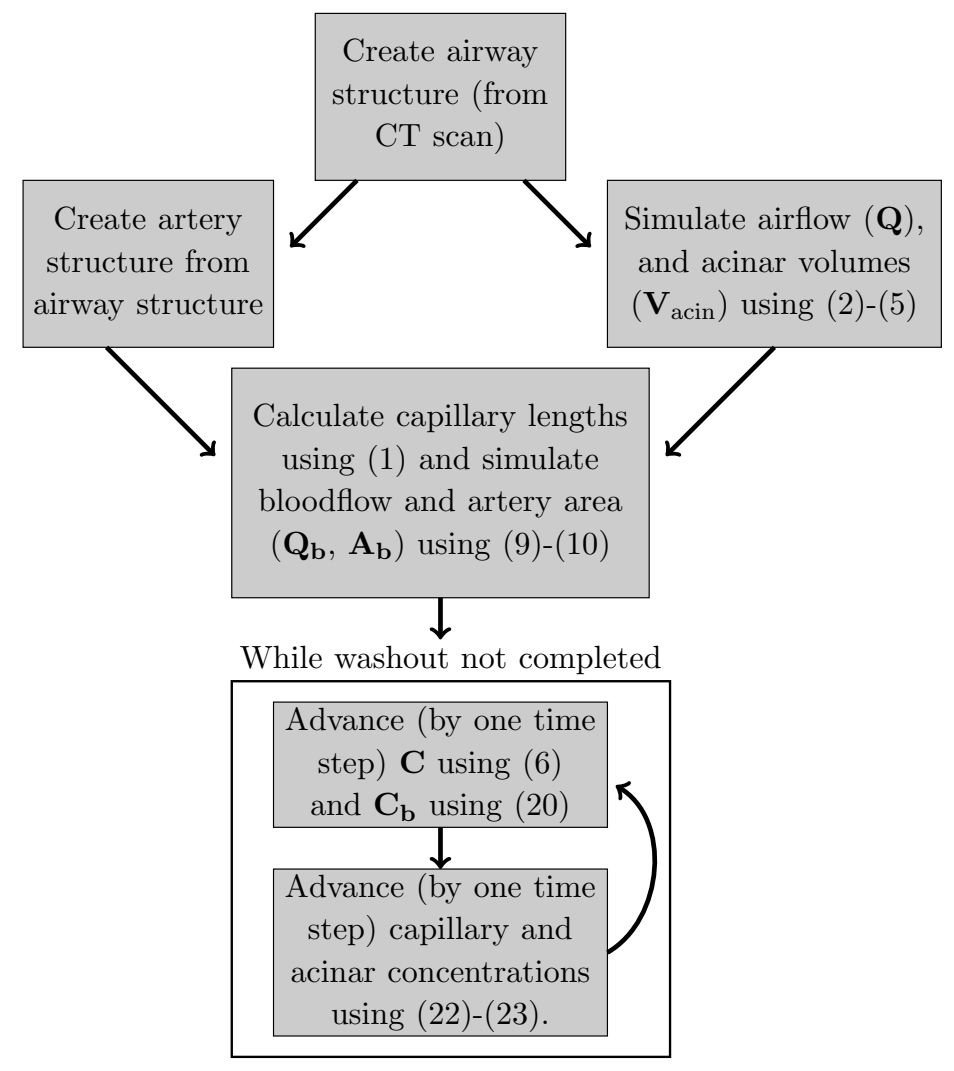

Figure 5: Flow diagram for the pulmonary model solution procedure.

in many applications gas transfer is simulated for a substantially longer period than blood flow, the solution speed is still dominated by the time taken to calculate the blood flow profile. This is because all other components of the model are solved implicitly, meaning time step sizes can be much larger $\left(\approx 10^{-2}\right)$ than that of the explicit blood flow scheme $\left(\approx 10^{-6}\right)$.

\section{Results}

\subsection{Clinical validation}

Given the complexity of the pulmonary model, a clear question to address is whether the model produces physically realistic, and clinically relevant results. Extremely detailed validation of the model throughout the entire pulmonary system is beyond the scope of a single study. Instead, we consider an array of experimental results within the literature. 
A clear validation of the airways ventilation and transport components of the model was presented by Foy et al. [20]. Within this work, the airways model was shown to be able to sensitively capture inter-patient differences when simulating outputs from the multiple-breath washout (MBW). Pozin et al. [31] analysed a similar uni-directional, pressure-driven flow model (though coupled with a lung parenchyma model), showing accurate alignment with physical ventilation measurements.

Alongside this, the use of Navier-Stokes to simulate blood flow has undergone a variety of different experimental validations within the literature. In their initial presentation, Olufsen et al. [11] showed clear concordance between simulations of arterial blood flow, and experimental measurements. Other validations have been performed using full 3D simulation [32], with strong concordance between the 3D and 1D models [33, 34]. Effective validation has also been performed in capillary networks [35], though this compared steady-state distributions only.

Convection-based models of oxygen transport in blood, similar to equation (20) (though also accounting for haemoglobin binding) have also been applied within the literature, showing strong concordance with experimental measurements [36].

The wealth of prior validation of the individual components of the pulmonary model gives us confidence in its ability to meaningfully capture the dominant mechanics of the pulmonary system. Clearly there would be value to validation of the model in a combined, holistic setting. We see this as a clear avenue for future research.

\subsection{Comparing different washout gases}

To illustrate the potential value of efficient pulmonary simulation, we apply the model to a clinically relevant question concerning the multiplebreath washout [37]. The MBW is a pulmonary function test that uses measurements of ventilation heterogeneity at the mouth, to make inferences about the presence of lung disease. Typically the test is performed in one of two ways; either air mixed with an inert-gas (such as $\mathrm{SF}_{6}$ of ${ }^{3} \mathrm{He}$ ) is washedin by the subject (breathed in until it is assumed to have equilibrated in the lungs), and then washout of the inert gas is performed by switching the subject back to an air mixture without the gas; or in the case of nitrogen washouts, since $\mathrm{N}_{2}$ is already present in air, no wash-in is performed, and instead a washout is performed using pure oxygen. 
Despite a wide variety of clinical $[38,39,40]$ and computational $[3,20]$ studies of the inert-gas washout, there is a lack of clear understanding as to how the choice of tracer gas may affect outputs [37]. Some recent studies have investigated this phenomenon [39], showing a potential bias between different gases. However, this finding hasn't been illustrated broadly enough to allow for consensus.

To help address this gap, we apply the pulmonary model to simulate the effect of tracer gas choice on MBW outputs, using the three most common tracer gases $\left(\mathrm{SF}_{6},{ }^{3} \mathrm{He}\right.$, and $\left.\mathrm{N}_{2}\right)$. Simulations were performed using each of the 26 patient-based lung structures. Each simulation was performed in an orthostatic position, after artificially washing in the tracer gas, at a concentration of $4 \%$ for $\mathrm{SF}_{6}$ and ${ }^{3} \mathrm{He}$, and $78 \%$ for $\mathrm{N}_{2}$. Diffusion rates for each gas are given in Table 1 , and for $\mathrm{SF}_{6}$ and ${ }^{3} \mathrm{He}, \mathcal{D}_{\text {cap }}$ was set to 0 . For each simulation we calculate the washout indices LCI (Lung Clearance Index) and $s_{\text {cond }}$, with precise definitions of each given in prior studies in the literature $[2,40]$.

As an initial step, in Figures 6 and 7 we present flow, cross-sectional area, and $\mathrm{N}_{2}$ concentration profiles at various locations throughout the pulmonary system, in a healthy lung structure. Considering the blood flow profile, we see small alternations in peak flow values every four heartbeats, due to the expansion and contraction of the capillaries in response to the alveoli. We also note that the arterial area profile behaves similarly to the flow profile, but has a slight offset of a few fractions of a second.

Considering Figure 7 no immediately obvious effect of the bloodstream can be seen in the tracheal $\mathrm{N}_{2}$ concentration profile. This is to be expected though, given that the individual contributions from the capillaries will be smoothed out by the time they reach the trachea. We see expected behaviour in the left pulmonary artery gas concentration profile, with an offset time of just over 60 seconds, corresponding to the imposed mean circulation time. Interestingly, the pulmonary artery empties at a much slower rate than the shown capillary. This is due to accumulation effects, and that flow in the capillaries is not perfectly synchronous (similar to the acinar regions). Finally, we note that before the washout begins, gas concentrations in the capillaries stabilise to just under $1 \%$, while concentrations in the acinus stabilised at $78 \%$. This is in line with the normal physiological concentration of nitrogen in the bloodstream, when breathing standard air ( $78 \%$ nitrogen).

Within Figures 8 and 9, we compare simulated $s_{\text {cond }}$ and LCI values, using the three different tracer gases. Firstly, comparing the non-pulmonary 
A.

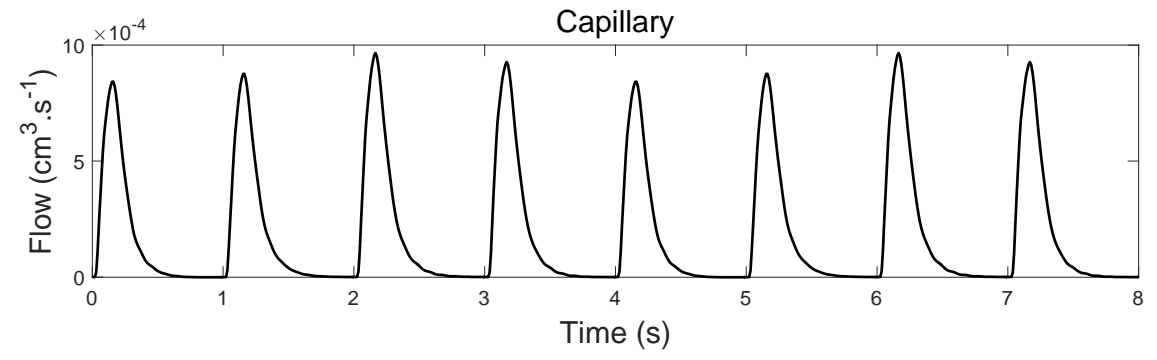

B.

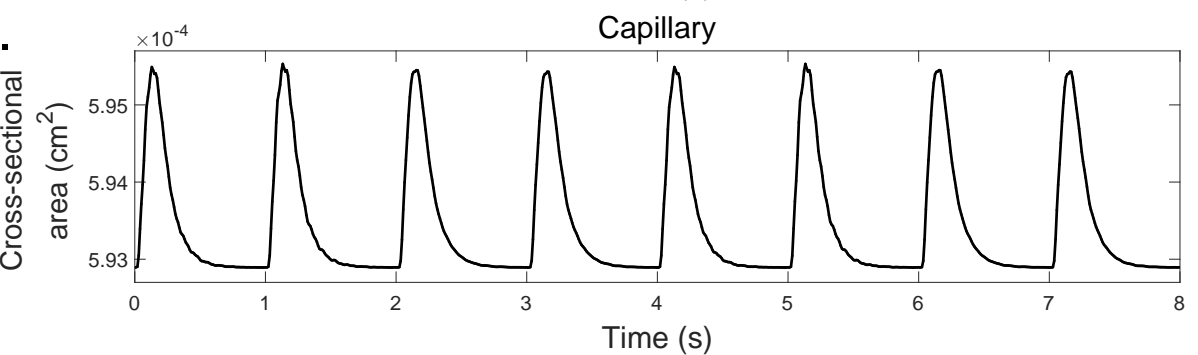

C.

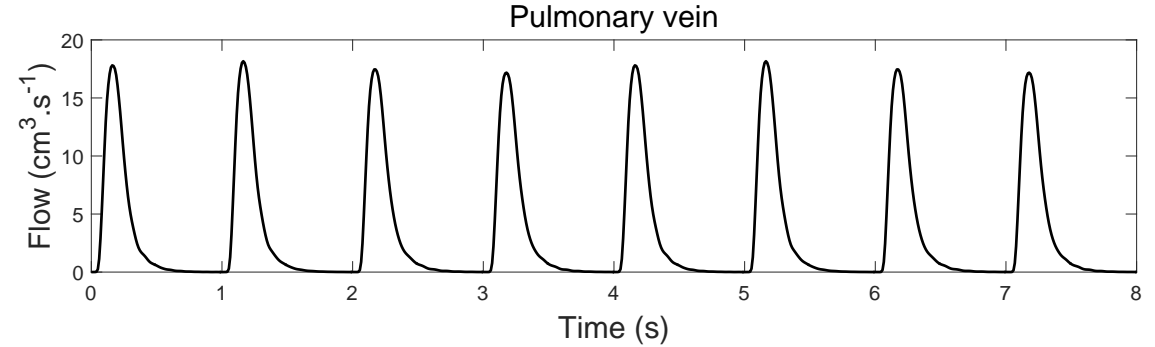

D.

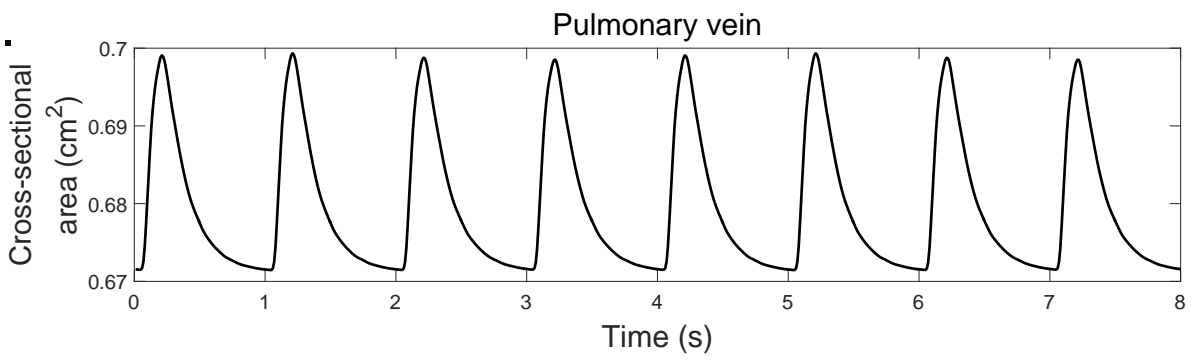

Figure 6: Blood flow and cross-sectional area profiles in a capillary $(A, B)$ and pulmonary vein $(\mathbf{C}, \mathbf{D})$. The capillaries undergo a strong sinusoidal pattern in peak values, driven by the expansion and contraction of the acinus. Flow is significantly larger in the pulmonary arteries, which also undergo a more significant cross-sectional area deformation.

diffusive gases, we see that there is a very slight negative bias in both LCI and $s_{\text {cond }}$ when using ${ }^{3} \mathrm{He}$. This is potentially due to the higher diffusivity of helium, which would allow constricted areas of the lung to washout slightly 


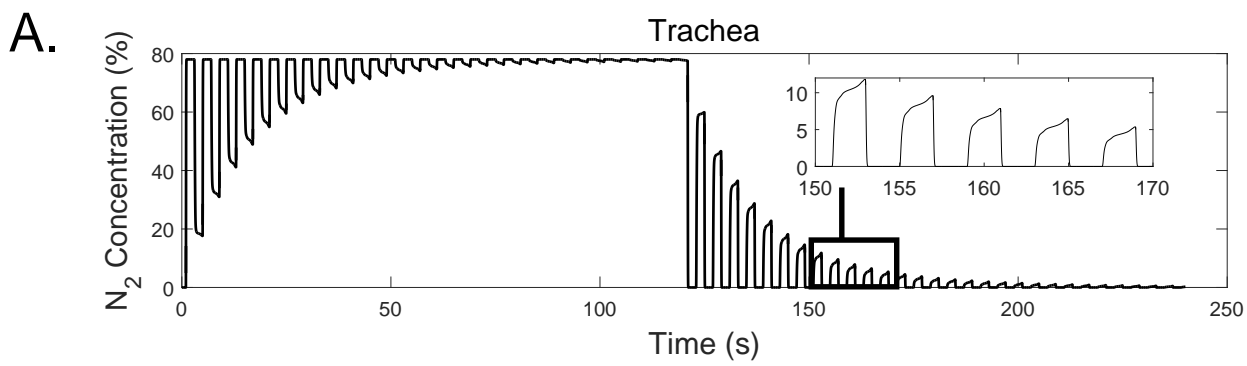

B.

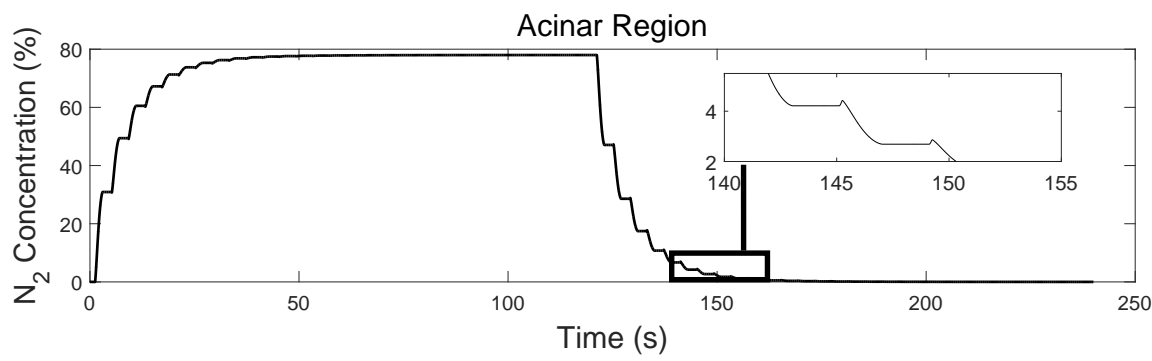

C.

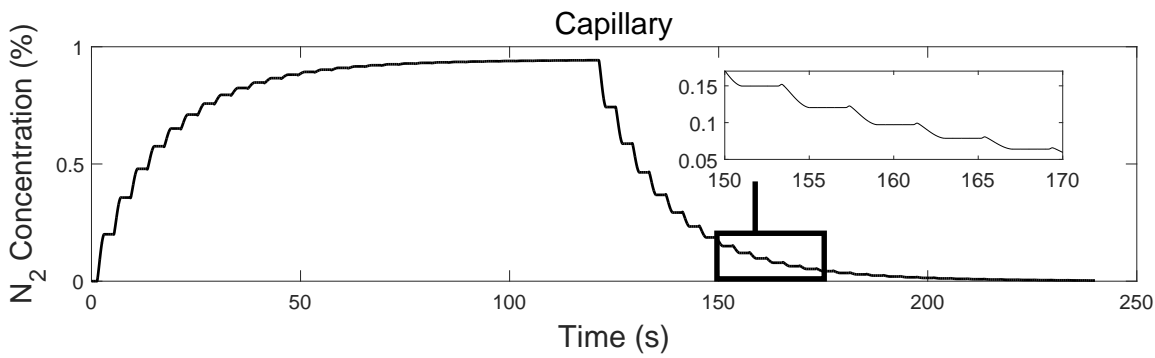

D.

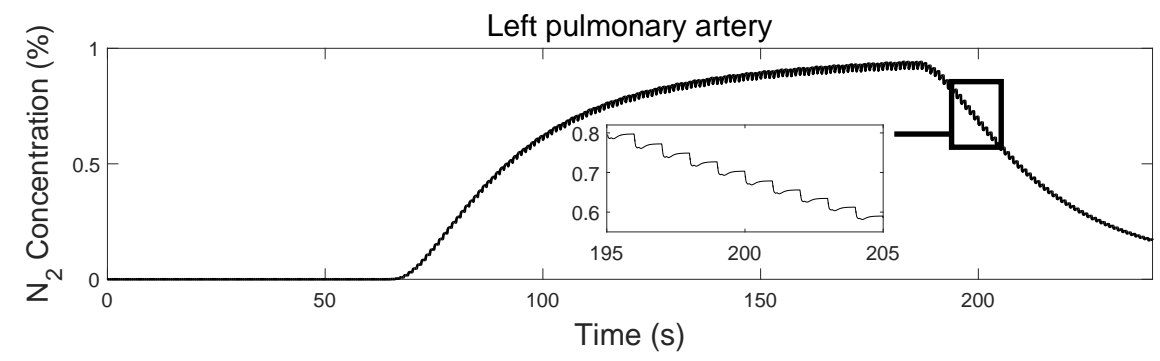

Figure 7: Concentration profiles at various locations in the pulmonary network. Nitrogen concentrations can be seen during wash-in and washout at the trachea (A), in an acinar region (B) and its associated capillary (C), and in the left pulmonary artery (D). For each washout profile, a zoom in is given to show behaviour over a few breaths.

faster. However, the confidence intervals for these differences greatly overlap 
zero, meaning the inference is quite weak.

A.

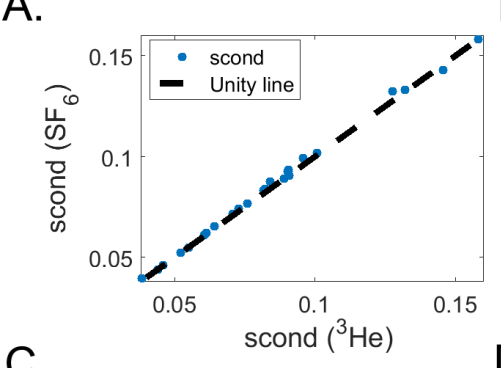

C.
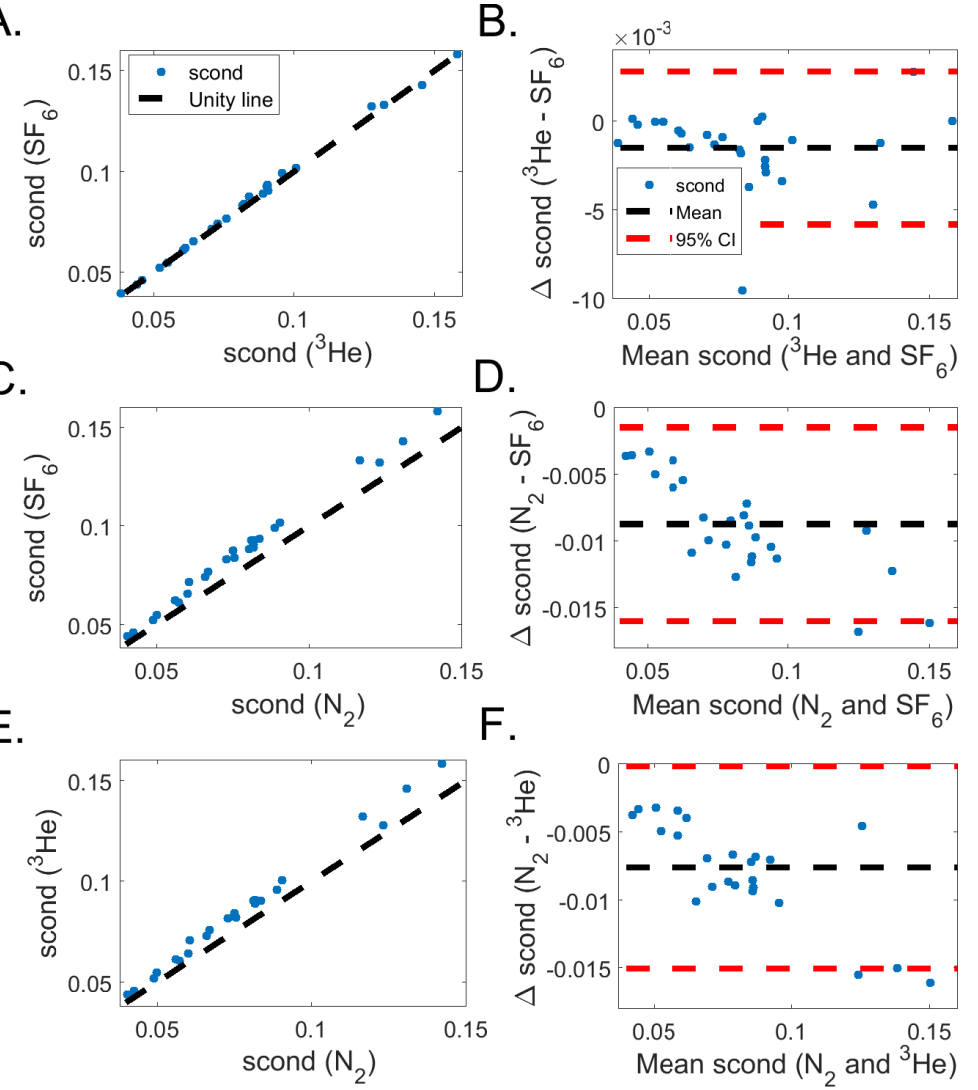

Figure 8: Comparison of simulated $s_{\text {cond }}$ with different tracer gases. Results comparing ${ }^{3} \mathrm{He}, \mathrm{SF}_{6}$ and $\mathrm{N}_{2}$ are shown, with a line of best fit (A,C,E) and Bland-Altman plots $(\mathrm{B}, \mathrm{D}, \mathrm{F})$ given.

Comparing $\mathrm{N}_{2}$ washouts to ${ }^{3} \mathrm{He}$ and $\mathrm{SF}_{6}$ washouts, the differences appear much more consistent. In both cases $s_{\text {cond }}$ has a noticeable negative bias, and LCI a positive bias, with magnitude of difference increasing with the index value. While small, this bias appears consistent, with $95 \%$ confidence intervals excluding zero.

\subsection{Error convergence}

To close the results section, we briefly illustrate error convergence of the model. Due to the model complexity, deriving analytical solution profiles is 
A.

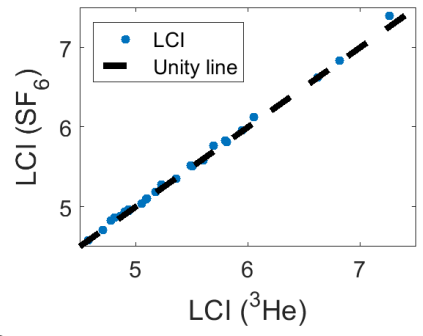

C.

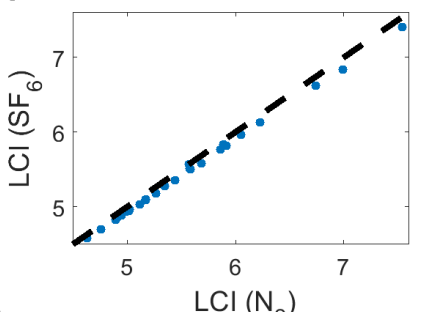

E.

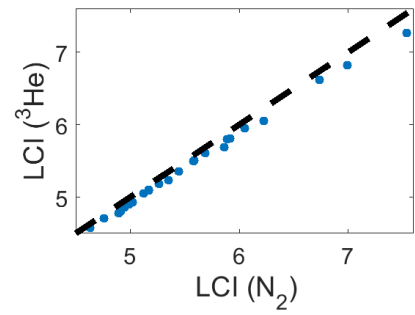

B.

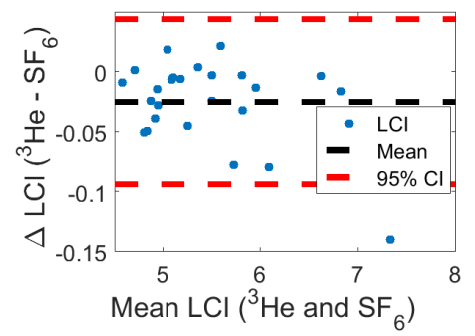

D.

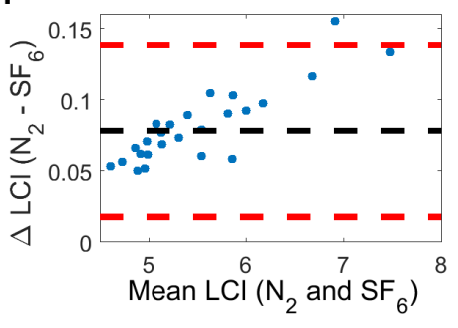

$\mathrm{F}$

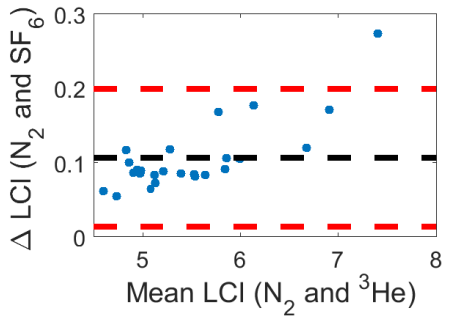

Figure 9: Comparison of simulated LCI with different tracer gases. Results comparing ${ }^{3} \mathrm{He}, \mathrm{SF}_{6}$ and $\mathrm{N}_{2}$ are shown, with a line of best fit $(\mathrm{A}, \mathrm{C}, \mathrm{E})$ and Bland-Altman plots $(\mathrm{B}, \mathrm{D}, \mathrm{F})$ given.

infeasible. Instead, we analyse error convergence of the model under simultaneous temporal and spatial refinement of the domains. We first define the error of a solution $\mathbf{x}$, relative to a fine mesh solution, $\mathbf{x}_{\text {fine }}$, such that

$$
\text { err }=\max \left|\frac{\mathbf{x}-\mathbf{x}_{\text {fine }}}{\mathbf{x}_{\text {fine }}}\right| .
$$

Note that to avoid catastrophic cancellation, solution error is only calculated at points where the elements of $\mathbf{x}_{\text {fine }}$ are sufficiently different from 0 .

Due to computational constraints, error was analysed in an artificially created 3 generation, and 5 generation pulmonary network, corresponding to a pulmonary structure with 28 and 78 branches respectively. Each generation 
started with a trachea of radius $1 \mathrm{~cm}$ and length $10 \mathrm{~cm}$. To grow the tree, at each bifurcation point, two new branches were created, at branch angles of $\pm 30^{\circ}$, with radius and branch length reduction factors of 0.8 and 0.6 . As an example, this leads to a branch length and radius of $6 \mathrm{~cm}$ and $0.6 \mathrm{~cm}$ at generation $2,4.8 \mathrm{~cm}$ and $0.36 \mathrm{~cm}$ at generation 3 , and so on. To add assymetry to the tree, each branch length and radius was multiplied by a normal-random number, drawn from the distribution $\mathcal{N}(1,0.05)$. The 3 generation and 5-generation airway tree structures are illustrated in Figure 10 .

Blood flow and cross-sectional area profiles (over 12 seconds) for the 3 generation network were calculated at the centre node of each branch (in arteries, capillaries and veins) with 1, 3, 7, 15, and 31 nodes per branch, and corresponding dt values of 0.002, 0.001,0.0005, 0.00025, 0.000125. Error was calculated as the maximum relative difference between the solution and the corresponding fine mesh solution at all branch bifurcations, and centre nodes. The fine mesh solution was created using 63 nodes per branch, and $d t=0.0000625$. A similar process was applied to analyse error in the 5 generation network, using $d t=0.0002,0.0001,0.00005,0.000025$ and $1,3,7$ and 15 nodes per branch, and a fine mesh solution created with 31 nodes per branch, and $d t=0.0000125$. The convergence of error in the structures can be seen in Figure 10. The convergence appears linear, as is expected from the Lax-Wendroff scheme.

A similar approach was applied to analyse convergence of the gas transport model. Simulations of a $30 \mathrm{~s} \mathrm{~N}_{2}$ washout (preceded by a $30 \mathrm{~s} \mathrm{~N}_{2}$ wash-in) were performed in a 3 and 5 generation network, with the same pairs of $d t$ and number of nodes as above. The discretisation was applied to both the blood flow and concentration model, across the airways and arterial network. The error convergence is shown in Figure 11, appearing linear as expected.

\section{Discussion}

We have presented the design and implementation of a gas transport model for the entire pulmonary system, alongside preliminary results from simulation of the multiple-breath nitrogen washout. Here we provide a brief discussion of clinical relevance, computational considerations, limitations, and potential future work. 

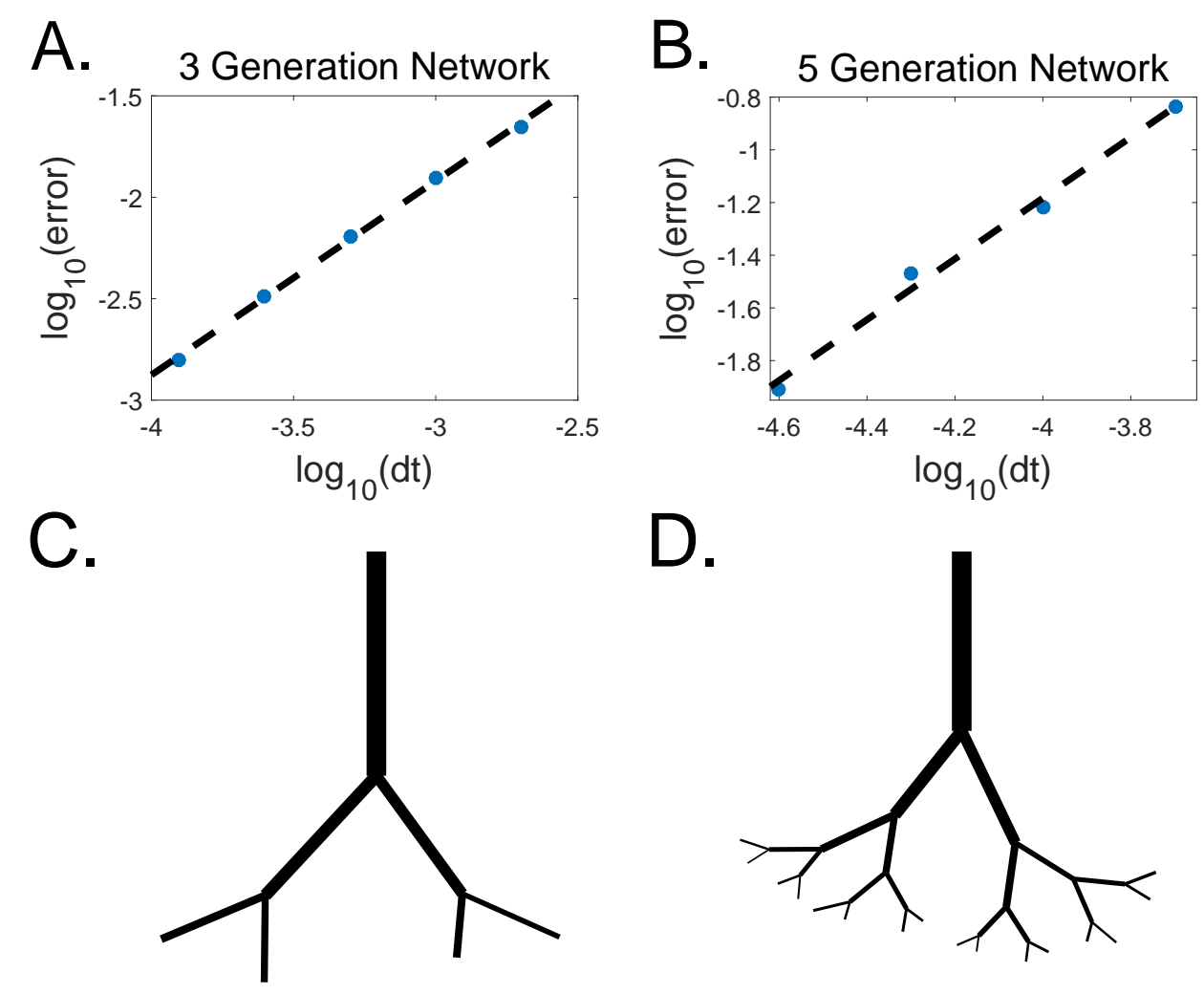

D.

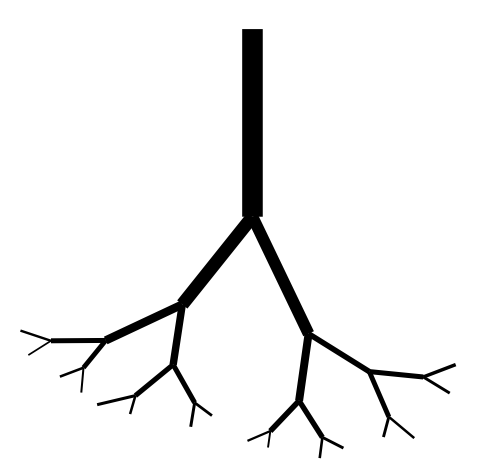

Figure 10: Error convergence of the blood flow model in a 3 generation network $(\mathrm{A}, \mathrm{C})$, and a 5 generation network $(\mathrm{B}, \mathrm{D})$. Both error plots have slopes of approximately 1, indicating linear convergence. Visualisation of the airway tree is also given, with line thickness corresponding to radius $(\mathrm{C}, \mathrm{D})$.

\subsection{Clinical relevance}

The results in Figures 8 and 9 suggest that the bloodstream interactions of nitrogen lead to a clear and consistent bias in washout indices; causing an elevation in LCI and reduction in $s_{\text {cond }}$. The positive bias of LCI using $\mathrm{N}_{2}$ confirms nicely with the clinical results of Jensen et al. [39] who showed that LCI was elevated when using $\mathrm{N}_{2}$, comparative to $\mathrm{SF}_{6}$, with the magnitude of difference increasing in the presence of cystic fibrosis. This result is further confirmed when comparing reference values for healthy controls, across various studies using different tracer gases [38]. However it should be noted that, to the best of our knowledge, no single prior study has illustrated this 

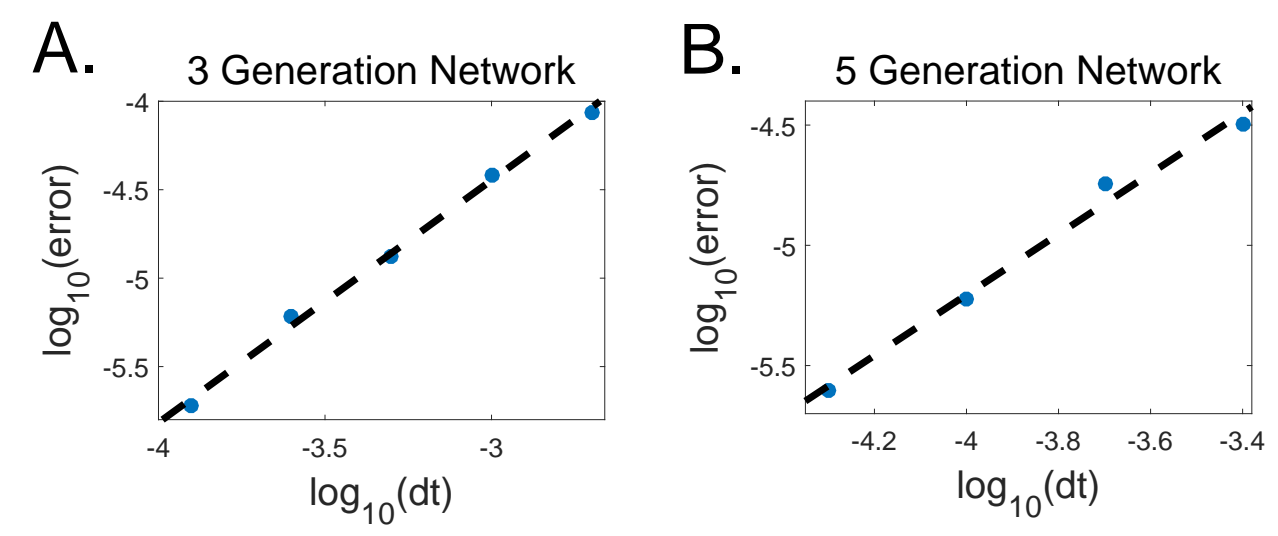

Figure 11: Error convergence of the pulmonary model in a 3 generation network (A), and a 5 generation network (B), over the arteries, acinar regions and airways. The two error plots have slopes of approximately 1.2-1.4, indicating linear convergence (as expected).

phenomenon within an asthmatic patient group. Thus, the results in this study help to provide evidence for the general nature of this phenomenon, beyond being specific to cystic fibrosis.

While there are some results in the literature comparing LCI, to the best of our knowledge, there are no large clinical studies which compare $s_{\text {cond }}$ under different gas choices. The results here suggest that the bias of $s_{\text {cond }}$ is opposite to that of LCI, with $\mathrm{N}_{2}$ leading to a reduction in the index, comparative to $\mathrm{SF}_{6}$, and ${ }^{3} \mathrm{He}$. Similar to LCI, this bias is quite small, however, it appears consistent, potentially allowing for correction when comparing clinical results obtained using different gases.

\subsection{Computational constraints}

As outlined previously, the computational scale of the model is quite large. To calculate the blood flow and arterial cross-sectional area profiles over 12 seconds, a system of approximately 1,500,000 variables was iteratively solved $10^{7}$ times. More generally, if we have an $N$ generation network, the number of branches can be approximated by $2^{N}$, leading to $2^{N+1}$ branches in the associated arterial network (excluding the capillaries). Assuming that only bifurcations occur (no trifurcations), and using the most coarse discretisation possible, each arterial branch contains 14 variables (two at the branch centre, 4 at each branch end, and 2 ghost nodes at each branch end). If each terminal bronchiole is subtended by a single acinus with a single capillary, there are 
$2^{N-1}$ total capillaries, each also having 14 variables. This leads to a total system complexity of

$$
\mathcal{O}\left(14\left(2^{N+1}+2^{N-1}\right)\right) .
$$

Further to this, the time step constraint on the Lax-Wendroff scheme scales linearly with the branch length (which directly relates to $\Delta x$ ). Assuming a branch length scaling factor of 0.6 (approximated from histological data [18]), as the tree grows, the total number of time steps scales according to $1.65^{N}$. For a 2 generation system, with a physically realistic geometry, we estimated a necessary step size of $\Delta t=10^{-3}$, leading to approximately $10^{4}$ steps per solution (of a 12 second blood profile). Given this, the total complexity of the model (expressed as number of necessary variable-iterations) is

$$
\mathcal{O}\left(14\left(2^{N+1}+2^{N-1}\right) \times 10^{4} 1.65^{N}\right) \approx \mathcal{O}\left(10^{5} \times 2^{1.72 N+1}\right) .
$$

For a full respiratory system $(N \approx 23)$, this would lead to approximately $10^{17}$ variable iterations. In practice this number is much greater, as beyond generation 16-17 the assumption of each branch being subtended by a single acinus fails to hold. Regardless, this presents significant computational strain, which we illustrate by comparing run times with and without GPU implementation, for various tree sizes (Figure 12).

\subsection{Limitations}

Models for pulmonary blood flow are not a completely recent development within the literature. However, most applications have been strongly limited by available computing power, and as such use simple approximations for flow in the capillaries and small arteries, and often exclude any venous component. As we have shown, through the embedding of the blood flow models in efficient parallel environments, many of these computational limitations may be overcome, allowing for effective simulation of the pulmonary system to much deeper levels.

Clearly though, this model still applies a variety of simplifying approximations, to make simulation at this depth computationally tractable. One of the strongest limitations of the model is the simplicity of the respiratory zone approximation. Representation of all veins and arteries below the structural resolution by a single capillary is clearly not physically realistic. However, this is primarily driven by a lack of realistic structural information and computational constraints. With access to more powerful HPC environments, 


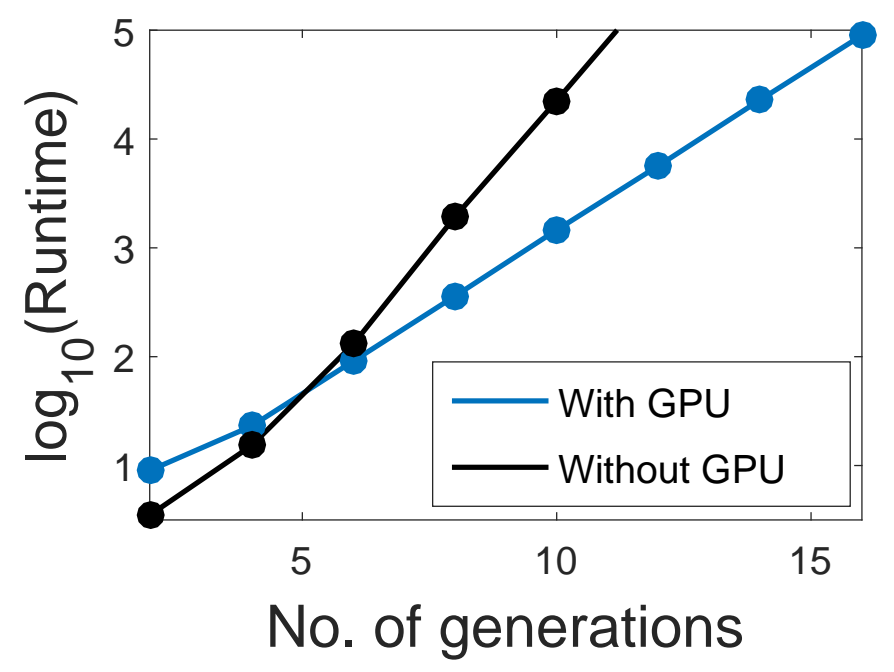

Figure 12: Comparison of runtimes when simulating blood flow with and without a GPU architecture. Run times are given for arterial trees with generation sizes $2-16$

and improvements in imaging techniques (such as micro-CT [41]), these limitations may be overcome.

Beyond this, the model is 1D in nature, meaning it cannot account for turbulent effects in both air and blood flow. Again, this choice is primarily made due to computational constraints. Given that both airflow and blood flow drastically slow as airway and artery radii decrease, we do not believe that the 1D approximation significantly affects accuracy. However, in the airflow and blood flow literature some groups have taken a multi-scale approach, whereby the largest branches are modelled using a full 3D Navier-Stokes, before transitioning to $1 \mathrm{D}$, past a certain point $[42,5]$.

Another limitation is the assumption of a linear stress-strain relationship between pressure and arterial cross-sectional area. Given the reduction in size and flow rates in the capillaries, the assumption of Newtonian flow may not be perfectly valid. This assumption was made to allow for tractability of the system. The investigation of tractable approaches to accounting for non-Newtonian flow behaviour in the capillaries is a clear avenue for future research.

Further to this, the model uses parameterised inflow and outflow boundary conditions for blood flow. While we have applied a simplistic model for 
inflow (Equation (19)), this could clearly be replaced with patient-specific data, or used to represent more complex scenarios (as would be caused by various heart conditions). Equally, the outlet boundary is parameterised by a Windkessel model, with 3 degrees of freedom $\left(\mathcal{R}_{1}, \mathcal{R}_{2}, \mathcal{C}_{T}\right)$, using values from a prior study in the literature [24]. However, in future work, investigations could be undertaken to improve the outlet boundary condition, and create more accurate estimations of downstream resistance and compliance.

Considering the arterial boundaries, the model for gas transfer also uses a simplistic approximation of gas circulation throughout the entire body, parameterised by a mean circulation time $t_{\text {circulation }}$. This is a simple approximations made to allow for closure of the model. More complex models that account for tissue absorption, and chemical reactions could be implemented if relevant experimental information was available.

Finally, the models were applied using pulmonary networks which were directly modelled based off of the CT-generated airway structures, as opposed to an independent CT-generated artery/vein network. While the pulmonary arteries and veins closely follow the airway structure, this is not a perfect correspondence. We note though that this does not represent a limitation of the model itself, which in the presented form may be applied to any given 1D airway and pulmonary structure.

While the outlined limitations may seem significant, they should be interpreted in context of the computational considerations. The majority of these limitations are not inherent to the model, but simply chosen factors (termination depths, etc.) based on computational constraints. Given the parallelisable nature of the model, advancements in computing power may allow many of these limitations to be overcome. The investigation of more complex and physically realistic approximations is a clear avenue for future work.

\subsection{Future work}

Within this study we have only illustrated the application of the pulmonary model to simulation of $\mathrm{N}_{2}$ gas transport. Adaptation of the model to a wider range of scenarios can be easily achieved though, with simple modifications.

The pulmonary model we have outlined is suitable for modelling transport of gases which cross the alveolar-capillary membrane, but which otherwise do not strongly interact with body tissues, or do so through a simple diffusive process. This means that the model in its current form cannot be 
applied to model the two gases of highest interest in respiration; oxygen and carbon dioxide. However, adaptation of the model into this scenario is fairly straightforward, by building on prior work in the literature, such as that of Ben-Tal [4]. For the simulation of oxygen transport, the alveolar-capillary exchange model can be modified to account for haemoglobin binding and saturation such that

$$
\frac{\partial V_{b} C_{b}}{\partial t}=\mathcal{D}_{\text {cap }}\left(P_{p, b}-P_{p, \text { acin }}\right)-N_{0} T_{h} V_{b} \frac{d S}{d t},
$$

where $C_{b}$ is the oxygen concentration, $N_{0}$ is the maximum number of molecules of $\mathrm{O}_{2}$ that can bind to haemoglobin, $T_{h}$ is the haemoglobin concentration, and $S(T)$ is the saturation function of oxygen in haemoglobin. Various different forms for the saturation function exist within the literature [4].

Ben-Tal [4] also presented similar models for transport of $\mathrm{CO}_{2}$ in the blood. However, to simulate both $\mathrm{O}_{2}$ and $\mathrm{CO}_{2}$ transfer across the pulmonary system, the model would need to be further expanded to incorporate a respiration model, which decomposes $\mathrm{O}_{2}$ into $\mathrm{CO}_{2}$. This could be incorporated by making the $\mathrm{O}_{2}$ and $\mathrm{CO}_{2}$ loss functions dependent on $\mathrm{CO}_{2}$ and $\mathrm{O}_{2}$ concentration, with parameters inferred from the aerobic respiration chemical reaction.

If these adaptations were applied to the model, it would potentially be capable of realistically simulating a much larger variety of physical scenarios, and to more precisely probe disease processes, and pulmonary function tests. The potential uses for such a model are quite vast, as there is still a strongly limited understanding of how different morphological changes across both the airway and veins/arteries affect the overall function of the pulmonary system. The model could be applied to variety of questions such as investigating how pulmonary arterial wall thickness increases (as can be seen due to high cholesterol) affect the $\mathrm{O}_{2}$ distribution across the lungs, or how an elevated heart rate may affect clinical respiratory measurements. This array of questions forms an exciting direction for future research within the field of computational modelling of the human body and the pulmonary system.

\section{Acknowledgements}

BF is funded by the Rhodes Trust.

This work was partially funded by an EU Framework 7 grant, through the AirPROM consortium. 


\section{Appendix A. Derivation of capillary length formula}

Within the methods, we present a formula for capillary length, relative to volume of the acinus it surrounds (Equation (1)). Here we derive this formula.

For computational tractability, we aim to approximate the capillary bed surrounding each acinus by a single capillary. For this to be a reasonable approximation, we need the mass of blood in a single capillary to approximate the mass of blood in the corresponding capillary bed. To achieve this, we assume that the single capillary wraps around the surface area of the acinus, covering a fraction $f$. This means that the capillary length is proportional to surface area, but also limited by its own diameter (which determines how much of the surface any piece of the capillary covers). Combining these two dependencies gives

$$
l_{\text {cap }}=f \frac{4 \pi R_{\text {acin }}}{2 R_{\text {cap }}^{0}}=f \frac{2 \pi R_{\text {acin }}}{2 R_{\text {cap }}^{0}} .
$$

The fraction $f$ of covered surface can be estimated by assuming a healthy adult lung $(\mathrm{FRC}=2.5 \mathrm{~L})$ is surrounded by $60 \mathrm{~mL}$ of capillary blood (a normal healthy adult value). Using a healthy lung from the Bordas set, this gives $f \approx 1 / 5$, meaning

$$
l_{\text {cap }}=\frac{2 \pi R_{\mathrm{acin}}^{2}}{5 R_{\mathrm{cap}}^{0}} .
$$

For this approximation to be appropriate, the ratio of lung volume to capillary blood should be independent of the number of generations in the airway tree. This is because this ratio significantly determines how strongly gas concentrations in the blood can affect concentrations in the airway (due to relative mass differences). To analyse whether this holds within our model, we first note that

$$
V_{\text {cap }}=l_{\text {cap }} \times A_{\text {cap }}=\frac{2}{5} \pi^{2} R_{\text {acin }}^{2} R_{\text {cap }}^{0}
$$

where $V_{\text {cap }}$ is the volume of the capillary, and $A_{\text {cap }}$ is its cross-sectional area.

For a structure with a given FRC, approximated using an $N$-generation network, we assume there is an average ratio between capillary volume and acinus volume, such that

$$
V_{\text {cap }, N} \approx k V_{\text {acin }, N},
$$


where the subscript $N$ denotes that the variable is from the $N$-generation network. If we take this structure, and add another generation, but hold FRC constant we have

$$
V_{\mathrm{acin}, N+1} \approx \frac{1}{2} V_{\mathrm{acin}, N},
$$

$$
R_{\mathrm{acin}, N} \approx\left(\frac{1}{2}\right)^{1 / 3} R_{\mathrm{acin}, N},
$$

as the number of acinar regions should approximately double in the new network.

If we assume that at each generation, the capillary and airway radii decrease with a branching factor $b_{f}$, we also have

$$
R_{\text {cap }, N+1} \approx b_{f} R_{\text {cap }, N},
$$

which means that the new capillary volume can be approximated as

$$
V_{\text {cap }, N+1} \approx \frac{2}{5} \pi^{2} b_{f}\left(\frac{1}{2}\right)^{2 / 3} R_{\mathrm{acin}, N}^{2} R_{\mathrm{cap}, N}^{0} .
$$

$$
V_{\text {cap }, N+1} \approx b_{f}\left(\frac{1}{2}\right)^{2 / 3} V_{\text {cap }, N} .
$$

If we assume the ratio between capillary volume and acinar volume is the same for both sized networks, we have that

$$
b_{f}\left(\frac{1}{2}\right)^{2 / 3}=\frac{1}{2},
$$

which means that the branching factor must be

$$
b_{f}=2^{-1 / 3} \approx 0.79 .
$$

This number is the well-known optimal branching factor [43] for the human lung, also known as the Hess-Murray law.

This illustrates that in an idealised branching network, this model holds, and accurately maintains an appropriate ratio (which we have set from standard physiological values as $2500 \mathrm{~L}: 60 \mathrm{~mL}$ ) between FRC and capillary blood volume. In an actual lung, average branching factors are usually slightly higher than the optimal factor, usually being around 0.81-0.86 [44]. Thus in 
practice for airway networks significantly larger or smaller than 16 generations, the surface area factor $f$ should be modified to maintain this ratio.

\section{Appendix B. Comparison of different alpha values}

The bloodflow model (equations (9)-(10)) contains a parameter $\alpha$, which accounts for the flow profile shape through the arterial cross-section. Results within this study have used the value $\alpha=1$, corresponding to a flat profile. For completeness, within Figure B.13 we compare flow and arterial area profiles using $\alpha=1.1$, a value commonly used within the literature [12] corresponding to a parabolic profile shape. As the figure shows, while $\alpha$ does influence overall behaviour, this change is fairly small, causing small shifts in peak values, and start times of the pulsatile behaviour. Given this, we do not believe that the choice of $\alpha=1$ significantly biases the results in this study.

A.

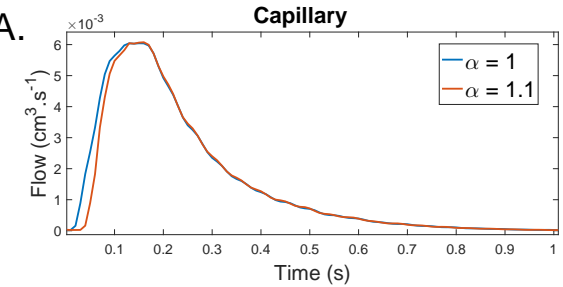

C.

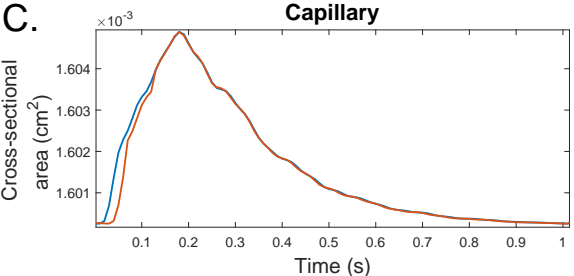

B.
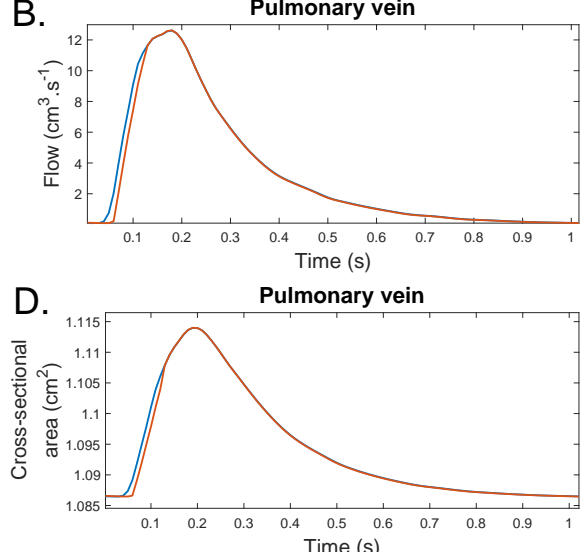

Figure B.13: Comparison of flow and area profiles using $\alpha=1$ and $\alpha=1.2$. As can be seen, the increase in $\alpha$ appears to only cause small shifts to overall profile behaviour in both peak flow and area values, and start time of the pulsatile behaviour. 
652

\section{Appendix C. Simplification of bifurcation equations}

To simplify the bifurcation equations (in the blood flow model), we first introduce notation for the variables:

$$
\begin{aligned}
& x_{1}=Q_{b, M}^{p, n+1}, \\
& x_{2}=Q_{b, M}^{p, n+1 / 2}, \\
& x_{3}=Q_{b, M+1 / 2}^{p, n+1 / 2}, \\
& x_{4}=Q_{b, 0}^{d_{1}, n+1} \text {, } \\
& x_{5}=Q_{b, 0}^{d_{1}, n+1 / 2} \text {, } \\
& x_{6}=Q_{b,-1 / 2}^{d_{1}, n+1 / 2} \text {, } \\
& x_{7}=Q_{b, 0}^{d_{2}, n+1} \\
& x_{8}=Q_{b, 0}^{d_{2}, n+1 / 2} \text {, } \\
& x_{9}=Q_{b,-1 / 2}^{d_{2}, n+1 / 2} \text {, } \\
& x_{10}=A_{b, M}^{p, n+1} \text {, } \\
& x_{11}=A_{b, M}^{p, n+1 / 2} \text {, } \\
& x_{12}=A_{b, M+1 / 2}^{p, n+1 / 2}, \\
& x_{13}=A_{b, 0}^{d_{1}, n+1} \text {, } \\
& x_{14}=A_{b, 0}^{d_{1}, n+1 / 2} \text {, } \\
& x_{15}=A_{b,-1 / 2}^{d_{1}, n+1 / 2} \text {, } \\
& x_{16}=A_{b, 0}^{d_{2}, n+1} \text {, } \\
& x_{17}=A_{b, 0}^{d_{2}, n+1 / 2} \text {, } \\
& x_{18}=A_{b,-1 / 2}^{d_{2}, n+1 / 2} \text {. }
\end{aligned}
$$

We then define

$$
\begin{aligned}
B_{M+1 / 2}(x) & =\frac{R_{b, M+1 / 2}^{0} f}{\rho_{b}} \sqrt{A_{b, M+1 / 2}^{0}}, \\
F_{M+1 / 2}\left(x_{1}, x_{2}\right) & =-\frac{2 \pi \nu_{b} R_{b, M+1 / 2}^{0}}{\delta} \frac{x_{1}}{x_{2}}, \\
\frac{d B_{M+1 / 2}}{d x}(x) & =\left(\frac{\partial B}{\partial R_{b}^{0}} \frac{d R_{b}^{0}}{d x}\right)_{M+1 / 2}^{n+1 / 2} \\
& =\left(2 \sqrt{x}\left(\sqrt{\pi} f R_{b}^{0}+\sqrt{A_{b}^{0}} \frac{d f}{d R_{b}^{0}}\right)-A \frac{d f}{d R_{b}^{0}}\right)_{M+1 / 2}\left(\frac{d R_{b, M+1 / 2}^{0}}{d x}\right), \\
\theta_{i} & =\frac{\Delta t}{\Delta x_{i}}, \\
\gamma & =\frac{\Delta t}{2} .
\end{aligned}
$$

Using this notation, we can rewrite Equations (13)-(18) in the form

$$
\mathbf{H}(\mathbf{X})=\mathbf{0}
$$

where $\mathbf{X}=\left(x_{1}, x_{2}, \ldots, x_{18}\right)^{T}$, and $\mathbf{H}=\left(h_{1}, h_{2}, \ldots, h_{18}\right)^{T}$. The vector $\mathbf{H}$ comprises the 18 residual functions, with the equations for conservation of 
flow (13):

$$
\begin{aligned}
& h_{1}=x_{1}-x_{4}-x_{7}, \\
& h_{2}=x_{2}-x_{5}-x_{8},
\end{aligned}
$$

the ghost node approximations (17)-(18):

$$
\begin{aligned}
h_{3,4,5} & =x_{2,5,8}-\frac{x_{3,6,9}}{2}+k_{1 a, 1 b, 1 c}, \\
h_{6,7,8} & =x_{11,14,17}-\frac{x_{12,15,18}}{2}+k_{2 a, 2 b, 2 c}, \\
k_{1 a} & =-\frac{Q_{b, M-1 / 2}^{p, n+1 / 2}}{2}, \\
k_{1 b} & =-\frac{Q_{b, 1 / 2}^{d_{1}, n+1 / 2}}{2}, \quad k_{1 c}=-\frac{Q_{b, 1 / 2}^{d_{2}, n+1 / 2}}{2}, \\
k_{2 a} & =-\frac{A_{b, M-1 / 2}^{p, n+1 / 2}}{2}, \\
k_{2 b} & =-\frac{A_{b, 1 / 2}^{d_{1}, n+1 / 2}}{2}, \quad k_{2 c}=-\frac{A_{b, 1 / 2}^{d_{2}, n+1 / 2}}{2}
\end{aligned}
$$

advancement of $A_{b}$ in time (15):

$$
\begin{aligned}
h_{9} & =x_{10}+\theta_{p} x_{3}-k_{3 a}, \\
h_{10,11} & =x_{13,16}-\theta_{d_{1}, d_{2}} x_{6,9}-k_{3 b, 3 c} \\
k_{3 a} & =A_{b, M}^{p, n}+\theta_{p} Q_{b, M-1 / 2}^{p, n+1 / 2} \\
k_{3 b} & =A_{b, 0}^{d_{1}, n}-\theta_{d_{1}} A_{b, 1 / 2}^{d_{1}, n}, \quad k_{3 c}=A_{b, 0}^{d_{2}, n}-\theta_{d_{2}} A_{b, 1 / 2}^{d_{2}, n}
\end{aligned}
$$


continuity of pressure (14):

$$
\begin{aligned}
h_{12,13} & =\frac{k_{4 a}}{\sqrt{x_{10}}}-\frac{k_{4 b, 4 c}}{\sqrt{x_{13,16}}}+k_{5 a, 5 b}, \\
h_{14,15} & =\frac{k_{4 a}}{\sqrt{x_{11}}}-\frac{k_{4 b, 4 c}}{\sqrt{x_{14,17}}}+k_{5 a, 5 b}, \\
k_{4 a} & =\left(f_{M}^{p} \sqrt{A_{b, M}^{p}}\right), \\
k_{4 b} & =\left(f_{0}^{d_{1}} \sqrt{A_{b, 0}^{d_{1}}}\right), \quad k_{4 c}=\left(f_{0}^{d_{2}} \sqrt{A_{b, 0}^{d_{2}}}\right), \\
k_{5 a} & =-f_{M}^{p}+f_{0}^{d_{1}}, \quad k_{5 b}=-f_{M}^{p}+f_{0}^{d_{2}},
\end{aligned}
$$

654

and advancement of $Q_{b}$ in time (16):

$$
\begin{gathered}
h_{16}=k_{6 a}-x_{1}-\theta_{p}\left(\frac{x_{3}^{2}}{x_{12}}+B_{M+1 / 2}\left(x_{12}\right)\right)+ \\
\gamma\left(F_{M+1 / 2}\left(x_{3}, x_{12}\right)+\frac{d B_{M+1 / 2}}{d x}\left(x_{12}\right)\right) \\
=k_{6 a}-x_{1}+G_{1}\left(x_{3}, x_{12}\right) \\
h_{17,18}=k_{6 b, 6 c}-x_{4,7}+\theta_{d_{1}, d_{2}}\left(\frac{x_{6,9}^{2}}{x_{15,18}}+B_{-1 / 2}\left(x_{15,18}\right)\right)+ \\
\gamma\left(F_{-1 / 2}\left(x_{6,9}, x_{15,18}\right)+\frac{d B_{-1 / 2}}{d x}\left(x_{15,18}\right)\right) \\
=k_{6 b, 6 c}-x_{4,7}+G_{2,3}\left(x_{6,9}, x_{15,18}\right) \\
k_{6 a}=Q_{b, M}^{p, n}+\theta_{p} \mathcal{T}_{2, M-1 / 2}^{p, n+1 / 2}+\gamma \mathcal{S}_{2, M-1 / 2}^{p, n+1 / 2}, \\
k_{6 b}=Q_{b, 0}^{d_{1}, n}-\theta_{d_{1}} \mathcal{T}_{2,1 / 2}^{d_{1}, n+1 / 2}+\gamma \mathcal{T}_{2,1 / 2}^{d_{1}, n+1 / 2} \\
k_{6 c}=Q_{b, 0}^{d_{2}, n}-\theta_{d_{2}} \mathcal{T}_{2,1 / 2}^{d_{2}, n+1 / 2}+\gamma \mathcal{T}_{2,1 / 2}^{d_{2}, n+1 / 2}
\end{gathered}
$$

Equations (C.1)-(C.10) form a system of 18 non-linear equations, for 18 variables. However, we note that Equations (C.1)-(C.6) are linear in the variables. We can use this linearity to collapse the system significantly.

Given we are solving $h_{i}=0$, (for $i=1 \ldots 18$ ), we first rearrange Equation 
(C.3) for $x_{2}, x_{5}$ and $x_{8}$, and substitute into Equation (C.2), to give (after rearrangement),

$$
\begin{aligned}
h_{a 1} & =x_{3}-x_{6}-x_{9}+k_{7}=0, \\
k_{7} & =k_{1 a}-k_{1 b}-k_{1 c} .
\end{aligned}
$$

We then rearrange Equations (C.5) and (C.6) for $x_{10}, x_{13}$ and $x_{16}$, and substitute into Equation (C.7), to give

$$
h_{a 2, a 3}=\frac{k_{4 a}}{\sqrt{k_{3 a}-\theta_{p} x_{3}}}-\frac{k_{4 b, 4 c}}{\sqrt{k_{3 b, 3 c}+\theta_{d_{1}, d_{2}} x_{6,9}}}+k_{5 a, 5 b}=0 .
$$

Equations (C.11) and (C.12) form a system of 3 non-linear equations with 3 unknowns. These can be solved for $x_{3}, x_{6}$ and $x_{9}$ using vector Newton method iterations, of the form

$$
\mathbf{X}_{a}^{i+1}=\mathbf{X}_{a}^{i}-\mathcal{J}_{a}^{-1} \mathbf{H}_{a}\left(\mathbf{X}_{a}^{i}\right),
$$

where $\mathbf{X}_{a}=\left(x_{3}, x_{6}, x_{9}\right)^{T}$ and $\mathbf{H}_{a}=\left(h_{a 1}, h_{a 2}, h_{a 3}\right)^{T}$. $\mathcal{J}_{a}$ is the Jacobian matrix of $\mathbf{H}_{a}$ and can be computed as

$$
\mathcal{J}_{a}=\left[\begin{array}{ccc}
1 & -1 & -1 \\
\xi_{1} & \xi_{2} & 0 \\
\xi_{1} & 0 & \xi_{3}
\end{array}\right]
$$

where

$$
\begin{aligned}
\xi_{1} & =\frac{1}{2} k_{4 a} \theta_{p}\left(k_{3 a}-\theta_{p} x_{3}\right)^{-3 / 2}, \\
\xi_{2,3} & =\frac{1}{2} k_{4 b, 4 c} \theta_{d_{1}, d_{2}}\left(k_{3 b, 3 c}+\theta_{d_{1}, d_{2}} x_{6,9}\right)^{-3 / 2} .
\end{aligned}
$$

Due to the small size of the system, the Jacobian inverse-product against $\mathbf{H}_{a}$ can be calculated explicitly, though is only updated once each time step.

This system collapse allows us to efficiently solve for $x_{3}, x_{6}$ and $x_{9}$. By substituting these values into Equations (C.3), (C.5) and (C.6) we can also easily calculate $x_{2}, x_{5}, x_{8}, x_{10}, x_{13}$, and $x_{16}$.

To solve for the remaining variables we first rearrange Equations (C.4) 
${ }_{671}$ for $x_{11}, x_{14}$, and $x_{17}$ and substitute into Equations (C.8) to give

$$
h_{b 1, b 2}=\frac{k_{4 a}}{\sqrt{\frac{x_{12}}{2}+k_{2 a}}}-\frac{k_{4 b, 4 c}}{\sqrt{\frac{x_{15,18}}{2}+k_{2 b, 2 c}}}+k_{5 a, 5 b}=0 .
$$

We then rearrange Equations (C.9) and (C.10) for $x_{1}, x_{4}$, and $x_{7}$, and substitute into Equation (C.1) to give

$$
\begin{aligned}
h_{b 3} & =k_{8}+G_{1}\left(x_{3}, x_{12}\right)-G_{2}\left(x_{6}, x_{15}\right)-G_{3}\left(x_{9}, x_{18}\right)=0 \\
k_{8} & =k_{6 a}-k_{6 b}-k_{6 c} .
\end{aligned}
$$

672 Since we have already solved for $x_{3}, x_{6}$, and $x_{9}$, Equations (C.13) and (C.14) 673 form a non-linear system with 3 unknowns. As with the first system, we solve 674 these equations for $x_{12}, x_{15}$, and $x_{18}$ using vector Newton method iterations 675 of the form

$$
\mathbf{X}_{b}^{i+1}=\mathbf{X}_{b}^{i}-\mathcal{J}_{b}^{-1} \mathbf{H}_{b}\left(\mathbf{X}_{b}^{i}\right),
$$

676 where $\mathbf{X}_{b}=\left(x_{12}, x_{15}, x_{18}\right)^{T}, \mathbf{H}_{b}=\left(h_{b 1}, h_{b 2}, h_{b 3}\right)^{T}$ and

$$
\mathcal{J}_{b}=\left[\begin{array}{ccc}
\xi_{1} & \xi_{2} & 0 \\
\xi_{1} & 0 & \xi_{3} \\
\xi_{4} & \xi_{5} & \xi_{6}
\end{array}\right]
$$


is the Jacobian of $\mathbf{H}_{b}$, with

$$
\begin{aligned}
\xi_{1}= & -\frac{1}{4}\left(\frac{x_{12}}{2}+k_{2 a}\right)^{-3 / 2}, \\
\xi_{2,3}= & \frac{1}{4}\left(\frac{x_{15,18}}{2}+k_{2 b, 2 c}\right)^{-3 / 2}, \\
\xi_{4}= & \frac{\partial G_{1}}{\partial x_{12}}, \\
= & -\theta_{p}\left(-\left(\frac{x_{3}}{x_{12}}\right)^{2}+\frac{1}{2} f R_{b, M+1 / 2}^{0} \sqrt{A_{b, M+1 / 2}^{0}} x_{12}^{-3 / 2}\right)+ \\
& \gamma\left(\frac{2 \pi R_{b, M+1 / 2}^{0}}{\delta} \frac{x_{3}}{x_{12}^{2}}-\frac{1}{2 x_{12}} \frac{d B_{M+1 / 2}}{d x}\left(x_{12}\right)\right) \\
\xi_{5,6}= & \frac{\partial G_{2,3}}{d x_{15,18}},\left(-\left(\frac{x_{6,9}}{x_{15,18}}\right)^{2}+\frac{1}{2} f R_{b,-1 / 2}^{0} \sqrt{A_{b,-1 / 2}^{0}} x_{15,18}^{-3 / 2}\right)+ \\
= & \left.-\theta_{d_{1}, d_{2}\left(\frac{1}{\delta}\right.} \frac{d B_{-1 / 2}\left(x_{15,18}\right)}{d x}\right) \\
& \gamma\left(\frac{2 \pi R_{b,-1 / 2}^{0}}{x_{15,18}}-\frac{1}{2 x_{15,18}}\right)
\end{aligned}
$$

As with the other $3 \times 3$ system, the size allows for efficient direct calculation of the Jacobian inverse-product against $\mathbf{H}_{b}$. Once a convergent solution (to within a desired tolerance) is reached, all remaining unsolved variables in $\mathbf{X}$ can be solved through simple substitutions into the remaining equations of System (C.1)-(C.10).

\section{Funding disclosures}

BF is funded by the Rhodes Trust.

Creation of the virtual airway structures was performed prior to this study, and was funded partially through the EU FP 7 AirPROM project.

Figure 1 was created with the assistance of Ms Sanjana Sharma.

\section{References}

[1] R. Bordas, C. Lefevre, B. Veeckmans, J. Pitt-Francis, C. Fetita, C. E. Brightling, D. Kay, S. Siddiqui, K. S. Burrowes, Development and anal- 
ysis of patient-based complete conducting airways models, PLoS One 10 (12) (2015) e0144105.

[2] B. H. Foy, D. Kay, R. Bordas, Modelling responses of the inert-gas washout and mri to bronchoconstriction, Respiratory Physiology \& Neurobiology 235 (2017) 8-17.

[3] J. H. Mitchell, E. A. Hoffman, M. H. Tawhai, Relating indices of inert gas washout to localised bronchoconstriction, Respiratory Physiology \& Neurobiology 183 (3) (2012) 224-233.

[4] A. Ben-Tal, Simplified models for gas exchange in the human lungs, Journal of Theoretical Biology 238 (2) (2006) 474-495.

[5] C.-L. Lin, M. H. Tawhai, E. A. Hoffman, Multiscale image-based modeling and simulation of gas flow and particle transport in the human lungs, Wiley Interdisciplinary Reviews: Systems Biology and Medicine 5 (5) (2013) 643-655.

[6] K. Horsfield, F. G. Relea, G. Gumming, Diameter, length and branching ratios in the bronchial tree, Respiration Physiology 26 (3) (1976) 351356.

[7] M. H. Tawhai, P. Hunter, J. Tschirren, J. Reinhardt, G. McLennan, E. A. Hoffman, CT-based geometry analysis and finite element models of the human and ovine bronchial tree, Journal of Applied Physiology 97 (6) (2004) 2310-2321.

[8] K. S. Burrowes, T. Doel, C. Brightling, Computational modeling of the obstructive lung diseases asthma and copd, Journal of Translational Medicine 12 (2) (2014) S5.

[9] M. H. Tawhai, K. S. Burrowes, Modelling pulmonary blood flow, Respiratory Physiology \& Neurobiology 163 (1-3) (2008) 150-157.

[10] J. Tu, K. Inthavong, G. Ahmadi, Computational fluid and particle dynamics in the human respiratory system, Springer Science \& Business Media, 2012.

[11] M. S. Olufsen, C. S. Peskin, W. Y. Kim, E. M. Pedersen, A. Nadim, J. Larsen, Numerical simulation and experimental validation of blood 
flow in arteries with structured-tree outflow conditions, Annals of Biomedical Engineering 28 (11) (2000) 1281-1299.

[12] N. Smith, A. Pullan, P. J. Hunter, An anatomically based model of transient coronary blood flow in the heart, SIAM Journal on Applied Mathematics 62 (3) (2002) 990-1018.

[13] R. W. Glenny, H. T. Robertson, Fractal modeling of pulmonary blood flow heterogeneity, Journal of Applied Physiology 70 (3) (1991) 10241030 .

[14] G. S. Krenz, J. H. Linehan, C. A. Dawson, A fractal continuum model of the pulmonary arterial tree, Journal of Applied Physiology 72 (6) (1992) $2225-2237$.

[15] K. S. Burrowes, P. J. Hunter, M. H. Tawhai, Anatomically based finite element models of the human pulmonary arterial and venous trees including supernumerary vessels, Journal of Applied Physiology 99 (2) (2005) 731-738.

[16] W. Kang, M. H. Tawhai, A. R. Clark, R. C. Sá, E. T. Geier, G. K. Prisk, K. S. Burrowes, In silico modeling of oxygen-enhanced mri of specific ventilation, Physiological reports 6 (7) (2018) e13659.

[17] C. I. Fetita, F. Prêteux, C. Beigelman-Aubry, P. Grenier, Pulmonary airways: 3 -d reconstruction from multislice ct and clinical investigation, IEEE transactions on medical imaging 23 (11) (2004) 1353-1364.

[18] K. Horsfield, Morphometry of the small pulmonary arteries in man., Circulation Research 42 (5) (1978) 593-597.

[19] B. H. Foy, D. Kay, A computational comparison of the multiple-breath washout and forced oscillation technique as markers of bronchoconstriction, Respiratory physiology \& neurobiology 240 (2017) 61-69.

[20] B. H. Foy, S. Gonem, C. Brightling, S. Siddiqui, D. Kay, Modelling the effect of gravity on inert-gas washout outputs, Physiological reports 6 (10) (2018) e13709.

[21] T. Pedley, R. Schroter, M. Sudlow, The prediction of pressure drop and variation of resistance within the human bronchial airways, Respiration Physiology 9 (3) (1970) 387-405. 
[22] M. Paiva, Gas transport in the human lung, Journal of Applied Physiology 35 (3) (1973) 401-410.

[23] B. Dutrieue, F. Vanholsbeeck, S. Verbanck, M. Paiva, A human acinar structure for simulation of realistic alveolar plateau slopes, Journal of Applied Physiology 89 (5) (2000) 1859-1867.

[24] M. S. Olufsen, Modeling the arterial system with reference to an anestesia simulator, Ph.D. thesis, Roskilde Universitetscenter, Institut for Studiet af Matematik og Fysik samt deres Funktioner i Undervisning, Forskning og Anvendelser (1998).

[25] S. Sherwin, L. Formaggia, J. Peiro, V. Franke, Computational modelling of $1 \mathrm{~d}$ blood flow with variable mechanical properties and its application to the simulation of wave propagation in the human arterial system, International Journal for Numerical Methods in Fluids 43 (6-7) (2003) 673-700.

[26] K. Wesseling, J. Jansen, J. Settels, J. Schreuder, Computation of aortic flow from pressure in humans using a nonlinear, three-element model, Journal of Applied Physiology 74 (5) (1993) 2566-2573.

[27] T. R. Marrero, E. A. Mason, Gaseous diffusion coefficients, Journal of Physical and Chemical Reference Data 1 (1) (1972) 3-118.

[28] K. S. Saladin, C. M. Porth, Anatomy \& physiology, McGraw-Hill, 1998.

[29] R. Sander, Compilation of henry's law constants (version 4.0) for water as solvent., Atmospheric Chemistry \& Physics 15 (8).

[30] A. M. Katz, Physiology of the Heart, Lippincott Williams \& Wilkins, 2010 .

[31] N. Pozin, S. Montesantos, I. Katz, M. Pichelin, I. Vignon-Clementel, C. Grandmont, A tree-parenchyma coupled model for lung ventilation simulation, International journal for numerical methods in biomedical engineering 33 (11) (2017) e2873.

[32] A. D. Bordones, M. Leroux, V. O. Kheyfets, Y.-A. Wu, C.-Y. Chen, E. A. Finol, Computational fluid dynamics modeling of the human pulmonary arteries with experimental validation, Annals of biomedical engineering (2018) 1-16. 
[33] L. Grinberg, E. Cheever, T. Anor, J. R. Madsen, G. Karniadakis, Modeling blood flow circulation in intracranial arterial networks: a comparative $3 \mathrm{~d} / 1 \mathrm{~d}$ simulation study, Annals of biomedical engineering 39 (1) (2011) 297-309.

[34] E. Boileau, P. Nithiarasu, P. J. Blanco, L. O. Müller, F. E. Fossan, L. R. Hellevik, W. P. Donders, W. Huberts, M. Willemet, J. Alastruey, A benchmark study of numerical schemes for one-dimensional arterial blood flow modelling, International journal for numerical methods in biomedical engineering 31 (10) (2015) e02732.

[35] R. P. Mayo, J. Olsthoorn, D. S. Charnock-Jones, G. J. Burton, M. L. Oyen, Computational modeling of the structure-function relationship in human placental terminal villi, Journal of biomechanics 49 (16) (2016) $3780-3787$.

[36] L. Gagnon, A. F. Smith, D. A. Boas, A. Devor, T. W. Secomb, S. Sakadžić, Modeling of cerebral oxygen transport based on in vivo microscopic imaging of microvascular network structure, blood flow, and oxygenation, Frontiers in computational neuroscience 10 (2016) 82.

[37] P. D. Robinson, P. Latzin, S. Verbanck, G. L. Hall, A. Horsley, M. Gappa, C. Thamrin, H. G. Arets, P. Aurora, S. I. Fuchs, et al., Consensus statement for inert gas washout measurement using multiple-and single-breath tests, European Respiratory Journal 41 (3) (2013) 507522 .

[38] L. Kent, P. Reix, J. Innes, S. Zielen, M. Le Bourgeois, C. Braggion, S. Lever, H. Arets, K. Brownlee, J. Bradley, et al., Lung clearance index: evidence for use in clinical trials in cystic fibrosis, Journal of Cystic Fibrosis 13 (2) (2014) 123-138.

[39] R. Jensen, S. Stanojevic, K. Gibney, J. G. Salazar, P. Gustafsson, P. Subbarao, F. Ratjen, Multiple breath nitrogen washout: a feasible alternative to mass spectrometry, PloS one 8 (2) (2013) e56868.

[40] S. Verbanck, D. Schuermans, A. Van Muylem, M. Paiva, M. Noppen, W. Vincken, Ventilation distribution during histamine provocation, Journal of applied physiology 83 (6) (1997) 1907-1916. 
817

818

819

820

821

822

823

824

825

826

827

828

[41] H. D. Litzlbauer, K. Korbel, T. L. Kline, S. M. Jorgensen, D. R. Eaker, R. M. Bohle, E. L. Ritman, A. C. Langheinrich, Synchrotron-based micro-CT imaging of the human lung acinus, The Anatomical Record 293 (9) (2010) 1607-1614.

[42] L. Formaggia, J.-F. Gerbeau, F. Nobile, A. Quarteroni, On the coupling of 3D and 1D Navier-Stokes equations for flow problems in compliant vessels, Computer Methods in Applied Mechanics and Engineering 191 (6-7) (2001) 561-582.

[43] B. Mauroy, M. Filoche, E. Weibel, B. Sapoval, An optimal bronchial tree may be dangerous, Nature 427 (6975) (2004) 633.

[44] E. R. Weibel, Geometry and dimensions of airways of conductive and transitory zones, Springer, 1963. 